\title{
Heme oxygenase-1 in the forefront of a multi-molecular network that governs cell-cell contacts and filopodia-induced zippering in prostate cancer
}

\author{
Alejandra V Paez ${ }^{1}$, Carla Pallavicini ${ }^{2}$, Federico Schuster ${ }^{1}$, Maria Pia Valacco ${ }^{1}$, Jimena Giudice ${ }^{3}$, Emiliano G Ortiz ${ }^{1}$, Nicolás Anselmino ${ }^{1}$, \\ Estefania Labanca ${ }^{4}$, Maria Binaghi ${ }^{1}$, Marcelo Salierno ${ }^{1}$, Marcelo A Martí', Javier H Cotignola', Anna Woloszynska-Read ${ }^{5}$, \\ Luciana Bruno ${ }^{2}$, Valeria Levi ${ }^{1}$, Nora Navone ${ }^{4}$, Elba S Vazquez ${ }^{1}$ and Geraldine Gueron ${ }^{\star, 1}$
}

Prostate cancer ( $\mathrm{PCa}$ ) cells display abnormal expression of cytoskeletal proteins resulting in an augmented capacity to resist chemotherapy and colonize distant organs. We have previously shown that heme oxygenase 1 (HO-1) is implicated in cell morphology regulation in PCa. Here, through a multi 'omics' approach we define the HO-1 interactome in PCa, identifying HO-1 molecular partners associated with the integrity of the cellular cytoskeleton. The bioinformatics screening for these cytoskeletalrelated partners reveal that they are highly misregulated in prostate adenocarcinoma compared with normal prostate tissue. Under H0-1 induction, PCa cells present reduced frequency in migration events, trajectory and cell velocity and, a significant higher proportion of filopodia-like protrusions favoring zippering among neighboring cells. Moreover forced expression of HO-1 was also capable of altering cell protrusions in transwell co-culture systems of PCa cells with MC3T3 cells (pre-osteoblastic cell line). Accordingly, these effects were reversed under siHO. Transcriptomics profiling evidenced significant modulation of key markers related to cell adhesion and cell-cell communication under HO-1 induction. The integration from our omics-based research provides a four molecular pathway foundation (ANXA2/HMGA1/POU3F1; NFRSF13/GSN; TMOD3/RAl14/VWF; and PLAT/PLAU) behind $\mathrm{HO}-1$ regulation of tumor cytoskeletal cell compartments. The complementary proteomics and transcriptomics approaches presented here promise to move us closer to unravel the molecular framework underpinning HO-1 involvement in the modulation of cytoskeleton pathways, pushing toward a less aggressive phenotype in PCa.

Cell Death and Disease (2016) 7, e2570; doi:10.1038/cddis.2016.420; published online 29 December 2016

Prostate cancer $(\mathrm{PCa})$ is the most frequently diagnosed cancer in men aside from skin cancer. ${ }^{1}$ Although PCa has been well defined in regards to the mutational landscape, analysis at the proteome level of these genetic alterations is still understudied. Most of the functional information of the cancer-associated genes relies in the proteome, an exceptionally complex biological system involving several proteins that function through dynamic protein-protein interactions and post-translational modifications. ${ }^{2}$

Tumor development and progression are partly consequence of defects in mechanisms controlling cytoskeletal remodeling. ${ }^{3}$ Actin re-arrangement and attachment to focal adhesions at the leading edge of a migrating cell, generate the driving force necessary for movement. ${ }^{3}$ The loss of cell-cell adhesion enables cancer cells to dissociate from the primary tumor mass and changes in cell-matrix interaction allows the cells to invade the surrounding stroma. ${ }^{4}$ Higher grade prostate carcinoma has been associated with the loss of cell adhesion molecules at adherens junctions. ${ }^{5}$ Cell protrusive forces are partly regulated by the GTP-binding protein Rac. ${ }^{6}$ The delicate equilibrium between the cell pushing and pulling forces drive leading edge dynamics and cell migration. Interdigitating filopodia are vital for the proper alignment and establishment of the initial cell-cell adhesions ${ }^{7}$ This event is known as adhesion 'zippering'.

Heme oxygenase $1(\mathrm{HO}-1)$ is the rate-limiting enzyme in heme degradation. $.^{9,10} \mathrm{HO}-1$ is as a stress response protein and a critical mediator of cellular homeostasis. ${ }^{11}$ Although the role of HO-1 in cancer is controversial, ${ }^{12}$ we have shown that its pharmacologic or genetic upregulation is associated with a less aggressive phenotype in $\mathrm{PCa} .{ }^{13} \mathrm{HO}-1$ inhibits cell proliferation, migration and invasion, ${ }^{14}$ it impairs tumor growth and angiogenesis in vivo and downregulates the expression of target genes associated with inflammation. ${ }^{14,15} \mathrm{HO}-1$ is also implicated in the modulation of cellular adhesion in $\mathrm{PCa}$, upregulating $\mathrm{E}$-cadherin and $\beta$-catenin expression, and relocating them to the cell membrane,${ }^{13}$ favoring a more epithelial phenotype. However, it is yet unclear which are the HO-1 interactors and how it regulates cytoskeleton organization.

In this work, we undertook a proteomics study to build the $\mathrm{HO}-1$ interactome in $\mathrm{PCa}$. We showed that $\mathrm{HO}-1$ binds to key factors associated with the dynamics of the actin cytoskeleton and induces the remodeling of the actin filament architecture

\footnotetext{
${ }^{1}$ Department of Biological Chemistry, School of Sciences, FCEN, University of Buenos Aires, IQUIBICEN-CONICET, Buenos Aires, Argentina; ${ }^{2}$ Department of Physics, FCEN, University of Buenos Aires, IFIBA-CONICET, Buenos Aires, Argentina; ${ }^{3}$ Department of Cell Biology and Physiology, School of Medicine, University of North Carolina at Chapel Hill, Chapel Hill, NC, USA; ${ }^{4}$ Department of Genitourinary Medical Oncology, The University of Texas, MD Anderson Cancer Center, Houston, TX, USA and ${ }^{5}$ Pharmacology and Therapeutics Department, Roswell Park Cancer Institute, Buffalo, NY, USA

${ }^{*}$ Corresponding author: G Gueron, Department of Biological Chemistry, School of Sciences, FCEN, University of Buenos Aires, IQUIBICEN-CONICET, Intendente Guiraldes 2160-Ciudad Universitaria Pab 2, 2ºiso, CM1, Buenos Aires CP1428, Argentina. Tel/Fax: +(5411) 4788-5755; E-mail: ggueron@gmail.com

Received 24.10.16; accepted 11.11.16; Edited by G Raschella'
} 
at filopodia. Further we obtained RNA-sequencing (RNA-Seq) profiles and determined a significant alteration of cytoskeletal genes by HO-1 induction, establishing molecular pathways by which forced expression of $\mathrm{HO}-1$ leads to a more adhesive and less invasive phenotype, further supporting the antitumoral function in PCa.

\section{Results}

Proteomics profiling of $\mathrm{HO}-1$-interacting proteins in $\mathrm{PCa}$ cells. We have previously demonstrated $\mathrm{HO}-1$ implications on the maintenance of the epithelial cell morphology and architecture. ${ }^{13}$ We hypothesized that $\mathrm{HO}-1$ could be exerting a regulatory role through its interaction with cytoskeletonassociated proteins. For this purpose, we conducted an indepth mass spectrometry-based proteomics study. We constructed a recombinant FLAGHO-1 protein. PC3 cells were transiently transfected with $\mathrm{FLAGHO}-1$ or the respective controls and treated with $\mathrm{H}_{2} \mathrm{O}_{2}$. Immunoprecipitated protein complexes were subjected to LC-ESI MS/MS (Figure 1a). This approach rendered a list of 56 proteins (Table 1). We performed a protein interaction network, showing interconnectivity either by reported gene colocalization, genetic interactions, predicted functional relationship, shared protein domains or physical interaction (Figure 1b, right panel). In all, $53 \%$ of the HO-1 interactome showed physical interconnectivity. The gene ontology (GO) analysis revealed an enrichment of proteins associated with four main functions: DNA and chromatin, RNA metabolism (post-transcription, including splicing), actin and cytoskeleton proteins and other proteins (Figure 1b, left panel).

Crystallographic analysis of $\mathrm{HO}-1$-interacting proteins. To assess protein domain interaction of $\mathrm{HO}-1$ interactors, we obtained their reference amino-acid sequences. The crystallographies with the highest coverage and score were selected and introduced into the protein domain database (PFAM) (Supplementary Figure S1). Using the inferred domain-domain interactions, we restricted the analysis to those proteins presenting domain interconnectivity, obtaining a network with 28 nodes and 29 edges (Figure 1d). We could not predict domain interaction between $\mathrm{HO}-1$ and its interactome because $\mathrm{HO}-1$ is a small globular protein with only one reported domain. However, we successfully predicted domain interactions among several $\mathrm{HO}$-1-interacting proteins: LASP1 and TES through the LIM domain, TES and ARNTL2 through LIM and HLH domains and TRIM28 and SRP20 through PHD and RMM_1, (Figure 1d). The crystallographic analysis of protein domains further supports physical interconnectivity of $\mathrm{HO}-1$ interactor proteins.

Analysis of multiple microarray data sets for cytoskeletal $\mathrm{HO}-1$-interacting proteins. Given that $\mathrm{HO}-1$ modulates the cell adhesive properties and morphology, ${ }^{13}$ we focused on $\mathrm{HO}$-1-interacting proteins associated with the cytoskeleton organization. To address their relevance in $\mathrm{PCa}$, we searched the public cancer microarray database, Oncomine (http://www.oncomine.org) (Figure 2a). The expression profile for these genes showed significant dysregulation for most of the cytoskeletal interactors of $\mathrm{HO}-1$, in particular, upregulation for TMOD3, TOP1, CBX3, RCC1, NPM1 and downregulation for ANXA2, HSPB1, STAT3 and TES (fold change $\geqslant 1.5, P$-value $\leqslant 0.05$; Figure $2 b$ ). The meta-analysis combining data from the independent data sets showed that the above-mentioned dysregulated cytoskeletal genes for prostate adenocarcinoma versus normal prostate gland lie within the $25 \%$ of the most consistently high- or low-expressed genes across this comparison. Of note, TES and MKLN lie within the $1-9 \%$ of the lowest expressed genes in PCa versus normal tissue (Figure 2b).

We also used the cBioPortal for Cancer Genomics platform (www.cbioportal.org) to search for the most common genetic alterations for these cytoskeletal proteins in $\mathrm{PCa}$ (Figure 2c, left panel). Results show presence of mutations, amplifications and deletions for the cluster of cytoskeletal genes selected. Of note, amplification was the most frequent alteration across the data sets (Figure 2c, right panel).

PCa cell 2-D migratory patterns under forced expression of HO-1. To examine whether the association of HO-1 with proteins implicated in the integrity of the actin cytoskeleton had an impact on PCa cellular migratory trajectories, we evaluated quantitatively the motion of cells in a woundhealing assay. Cells were treated with hemin (specific inducer of $\mathrm{HO}-1,80 \mu \mathrm{M}, 24 \mathrm{~h}$ ). A linear scratch was done along the culture plate and cells were imaged using phase contrast

\footnotetext{
Figure 1 Construction of the HO-1 interactome in PCa cells. (a) Simplified schematic workflow of the construction of the HO-1 interactome in PC3 cells. FLAGHO-1 immunoprecipitation assays were performed from PC3 cell extracts that had been previously transiently transfected with FLAGHO-1 plasmid or empty FLAG vector and treated with $\mathrm{H}_{2} \mathrm{O}_{2}(200 \mu \mathrm{M}, 1 \mathrm{~h})$. For LC-ESI MS/MS analyses, peptides were desalted and concentrated using a C18 resin. Peptides were analyzed by reverse-phase chromatography before mass spectrometry analysis. The peak lists obtained were processed and analyzed with NCBI databases using Mascot Software (Matrix Science Inc, Boston, MA, USA) and compared with the human genome, with a fragment ion mass tolerance of $0.80 \mathrm{Da}$ and a parent ion tolerance of $2.0 \mathrm{Da}$. (b) Protein interaction network of HO-1 interactome in PC3 cells displaying interconnectivity either by reported gene colocalization, genetic interactions, predicted functional relationship, shared protein domains or physical interaction (left panel). Protein interaction network shows physical interconnectivity between HO-1-interacting proteins (right panel). (c) GO analysis of HO-1-interacting proteins was performed using DAVID software (https://david.ncifcrf.gov/). The resulting categories for biological process, cellular components and molecular functions were further subclustered into four global categories named: 'DNA and chromatin' (red), 'RNA processing' (blue), 'actin and cytoskeleton' (yellow) and 'other function' (green). (d) Schematic representation of the inferred domain-domain interactions of $\mathrm{HO}-1$-interacting proteins. Reference amino-acid sequences of protein domains of $\mathrm{HO}-1$ interactors were aligned against the Protein Data Bank (PDB). The crystallographies with the highest coverage and score were selected and introduced into the protein domain database (PFAM). HO-1 candidate interactors protein domains were annotated. Individual searches in the Interaction Protein Family (iPfam) database were performed. The networks were built using Cytoscape 3.2.1 software showing 201 nodes (rectangles) and 327 edges (black lines). The interactome network (framed in orange dashed-line, upper panel) was simplified and consists of 28 nodes (circles) and 29 edges (black lines) (bottom panel) representing: yellow circles, domains of HO-1-interacting proteins; red circles, HO-1-interacting proteins; light blue circles, other protein domains interacting with domains of HO-1-interacting proteins
} 
a

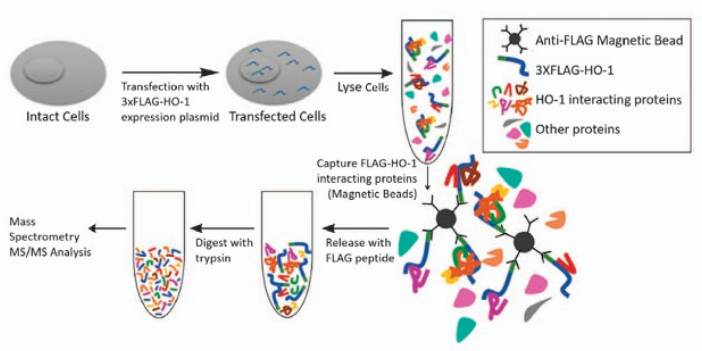

C
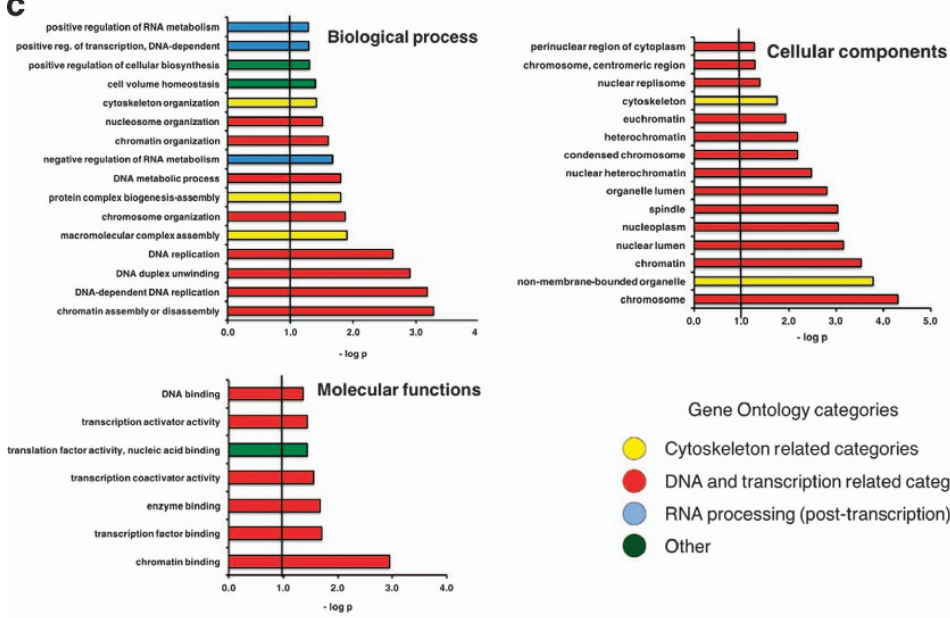

Gene Ontology categories

Cytoskeleton related categories

Other

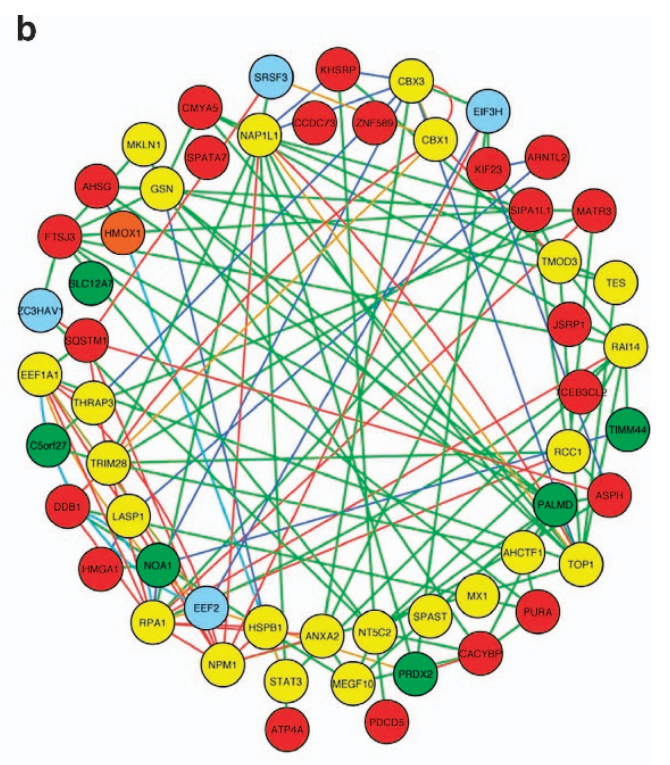

DNA and transcription related categories

RNA processing (post-transcription) related categories
Interaction key

- Genetic interactions

- Physical interactions

- Co-localization

- Pathway

- Predicted

- Shared protein domains

d
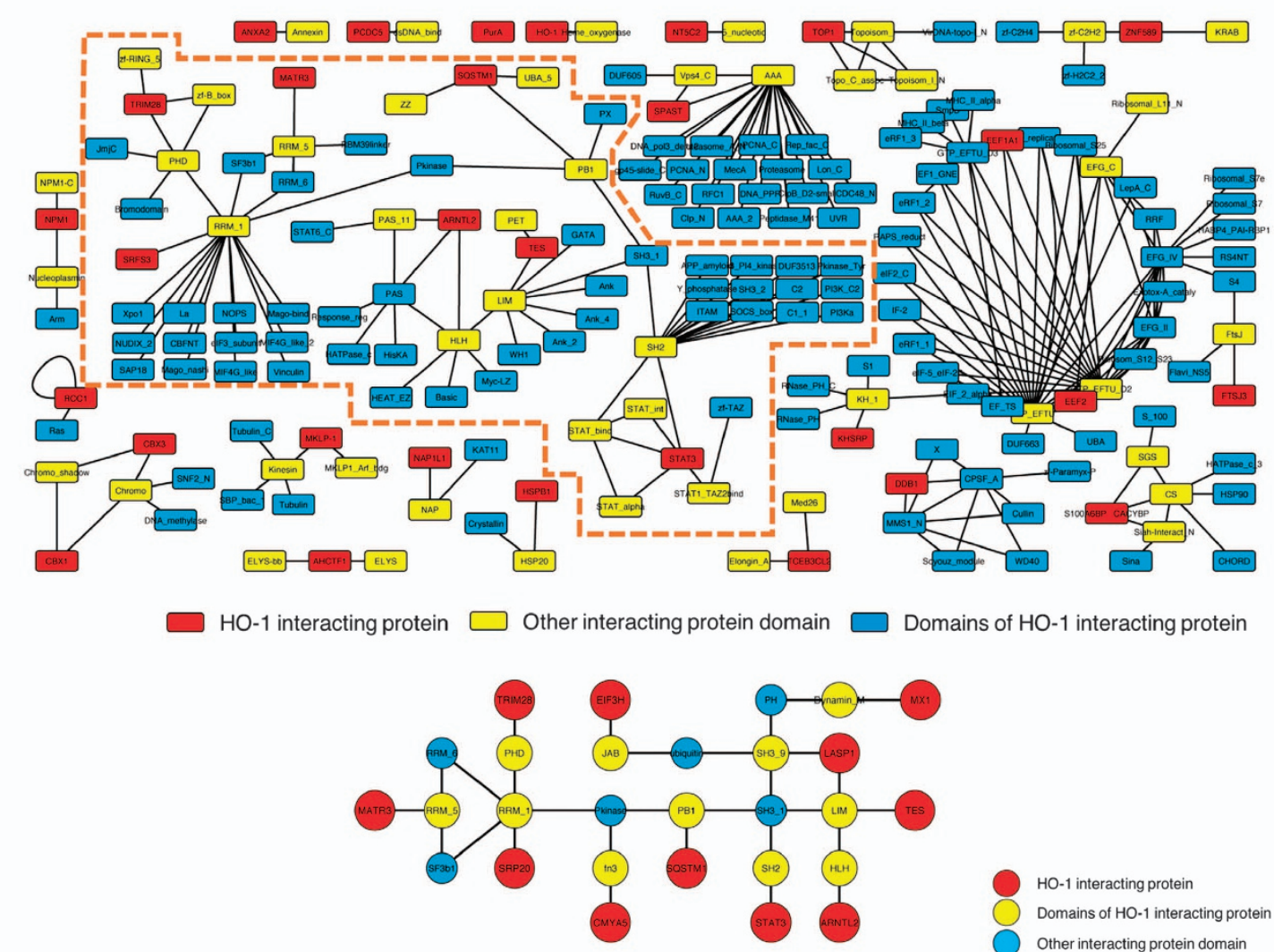

HO-1 interacting protein

Domains of HO-1 interacting protein

Other interacting protein domain 
Table 1 List of HO-1-interacting proteins identified by MS/MS analysis

\begin{tabular}{|c|c|c|c|c|}
\hline Protein name & $\begin{array}{l}\text { Gene } \\
\text { symbol }\end{array}$ & $\begin{array}{c}\text { Theroretical molecular } \\
\text { mass (Da) }\end{array}$ & $\begin{array}{l}\text { No. of } \\
\text { peptides }\end{array}$ & Coverage $\%$ \\
\hline High mobility group protein HMG-I/HMG-Y isoform a & AHCTF1 & 11669 & 2 & 29.2 \\
\hline Alpha-2-HS-glycoprotein & AHSG & 39193 & 5 & 25 \\
\hline Anexin 2 & ANXA2 & 38808 & 3 & 13.6 \\
\hline Cycle-like factor CLIF & ARNTL2 & 67908 & 1 & 2 \\
\hline Aspartyl beta-hydroxylase & ASPH & 85959 & 1 & 3 \\
\hline Potassium-transporting ATPase alpha chain 1 & ATP4A & 114045 & 1 & 2 \\
\hline Putative uncharacterized protein C5orf27 & C5ORF27 & 10849 & 1 & 14.58 \\
\hline Chromobox homolog 1 & CBX1 & 21519 & 2 & 11.2 \\
\hline Chromobox homolog 3 & CBX3 & 20997 & 2 & 15.2 \\
\hline Coiled-coil domain-containing protein 73 & CCDC73 & 1079 & 1 & 2.78 \\
\hline Cardiomyopathy-associated protein 5 & CMYA5 & 17545 & 1 & 0.9 \\
\hline Damage-specific DNA-binding protein $1,127 \mathrm{kDa}$ & DDB1 & 128086 & 1 & 1.4 \\
\hline Translation elongation factor 1 -alpha & EEF1A1 & 35825 & 2 & 12 \\
\hline Eukaryotic translation elongation factor 2 & EEF2 & 96246 & 1 & 2.9 \\
\hline Translation initiation factor elF3 p40 subunit & EIF3H & 40075 & 1 & 5 \\
\hline FtsJ3 protein & FTSJ3 & 84015 & 1 & 2 \\
\hline Gelsolin & GSN & 31052 & 3 & 22.7 \\
\hline High mobility group AT-hook 1 & HMGA1 & 34635 & 1 & 5.1 \\
\hline Heme oxygenase 1 & $\mathrm{HO}-1$ & 32798 & 5 & 25 \\
\hline Heat shock $27 \mathrm{kDa}$ protein & HSPB1 & 22427 & 3 & 31.4 \\
\hline Junctional sarcoplasmic reticulum protein 1 & JSRP1 & 36296 & 1 & 2 \\
\hline KH-type splicing regulatory protein & KHSRP & 10362 & 1 & 24.4 \\
\hline Lasp-1 & LASP1 & 30185 & 1 & 5 \\
\hline Matrin 3 & MATR3 & 95078 & 2 & 5 \\
\hline Multiple epidermal growth factor-like domains protein 10 & MEGF10 & 122121 & 1 & 2.11 \\
\hline Muskelin 1 & MKLN1 & 84713 & 11 & 17 \\
\hline Kinesin-like protein KIF23 & MKLP-1 & 98842 & 1 & 1.7 \\
\hline Interferon-induced Mx protein & MX1 & 75929 & 1 & 2 \\
\hline Nucleosome assembly protein 1-like 1 & NAP1L1 & 45631 & 1 & 4.1 \\
\hline Nitric oxide-associated protein & NOA1 & 78409 & 1 & 4.44 \\
\hline Nucleophosmin 1 & NPM1 & 3109 & 2 & 12.7 \\
\hline 5'-Nucleotidase, cytosolic II & NT5C2 & 65384 & 1 & 2 \\
\hline Palmdelphin, isoform CRA_a & PALMD & 53404 & 1 & 2 \\
\hline Programmed cell death 5 short isoform & PDCD5 & 4472 & 1 & 32 \\
\hline Peroxiredoxin 2 & PDX2 & 22014 & 1 & 5 \\
\hline Purine-rich element binding protein A & PURA & 35003 & 1 & 8.2 \\
\hline Retinoic acid-induced 14 & RAl14 & 110617 & 1 & 1.5 \\
\hline Regulator of chromosome condensation & RCC1 & 44485 & 1 & 3.7 \\
\hline Replication protein A 70 kDa DNA-binding subunit & RPA1 & 68723 & 1 & 3.6 \\
\hline Ribosomal protein SA pseudogene 9 & RPSA1 & 32947 & 1 & 9 \\
\hline s100 calcium- binding protein A6 & S100A6BP & 1023 & 1 & 8 \\
\hline Splicing factor, arginine/serine-rich 3 & SFRS3 & 19546 & 1 & 11.8 \\
\hline Signal-induced proliferation-associated 1-like protein 1 & SIPA1L1 & 201102 & 1 & 1.25 \\
\hline $\mathrm{K}-\mathrm{Cl}$ co-transporter KCC4 & SLC12A7 & 120327 & 1 & 0.6 \\
\hline Isoform 4 of Spastin & SPAST & 54385 & 1 & 7.23 \\
\hline Spermatogenesis-associated protein 7 & SPATA7 & 8799 & 1 & 36.84 \\
\hline Sequestosome 1 & SQSTM1 & 4837 & 1 & 4.5 \\
\hline RNA polymerase II transcription factor SIII subunit A3-like-2 & TCEB3CL2 & 59735 & 1 & 3.48 \\
\hline Testis-derived transcript & TES & 49789 & 1 & 3.1 \\
\hline Thyroid hormone receptor-associated protein 3 & THRAP3 & 108686 & 1 & 1.9 \\
\hline Mitochondrial import inner membrane translocase subunit & TIMM44 & 15215 & 1 & 19.55 \\
\hline Tropomodulin 3 & TMOD3 & 39727 & 2 & 9.7 \\
\hline DNA topoisomerase 1 & TOP1 & 66890 & 2 & 5.3 \\
\hline Tripartite motif-containing 28 & TRIM28 & 80621 & 6 & 18 \\
\hline Zinc-finger CCCH-type, antiviral 1 & ZC3HAV1 & 103135 & 3 & 7.3 \\
\hline Zinc-finger protein 589 & ZNF589 & 40736 & 7 & 36 \\
\hline
\end{tabular}

microscopy at 1 frame/20 min during $48 \mathrm{~h}$. Single-cell trajectories (Figure 3a) were analyzed to obtain the mean squared displacement (MSD) versus time plot in each experimental condition. Figure $3 \mathrm{~b}$ shows that the MSD obtained in both conditions increases as a function of time; however, after $10 \mathrm{~h}$, the MSD obtained in hemin-treated cells was significantly smaller than in control cells. HO-1 induction significantly reduces the area explored by $\mathrm{PCa}$ cells in the assayed temporal window compared with control cells.
$\mathrm{PCa}$ cellular protrusions under $\mathrm{HO}-1$ induction. To analyze whether there were variations in cell-cell interactions, we determined the distribution of the distances to the first neighboring cell under $\mathrm{HO}-1$ modulation (Figure $3 \mathrm{c}$ ). For every cell or cell cluster, we calculated the distance to all the other cells/cell clusters (white line) and to the first neighboring cell (red line) (Figure 3c). Results show that $\mathrm{HO}-1$ induction in PC3 cells significantly reduced the distances to the first neighboring cell $(P<0.05$; Figure $3 \mathrm{~d})$. 
We used confocal microscopy to study the changes in the cytoskeletal organization, in particular the cell adhesion zippering through the actin stress fibers at the leading edge level of PCa cells. To quantify contacts among cells, we selected regions in which the filopodia from two neighboring cells touched each other, considered as 'contacts', and divided these regions into segments where the distance between the cells remained constant (Figures $3 e$ and f). An intensity profile for each of these areas was analyzed with a custom made algorithm to count contacts (Figure 3e). A 'contact density' was defined for each region as the ratio between the number of contacts and the length of the profile. Results showed an increased contact density under $\mathrm{HO}-1$ induction, favoring adhesion zippering (Figure 3g; $P<0.05$ ). Filopodia-like protrusions (cell filopodia density) were also enhanced by hemin treatment (Figure $3 \mathrm{~h} ; \mathrm{P}<0.05$ ).

Using a specific siRNA for $\mathrm{HO}-1$ (siHO-1), the effects observed on the augmented filopodia per cell and cell-cell contact, under HO-1 induction, were reversed (Figure $4 \mathrm{a}$, left panel). Efficiency of HO-1 depletion was confirmed by western blot (Figure 4a, right panel). Accordingly cell zippering under $\mathrm{HO}-1$ induction, was also impaired (Figure 4b). Contacts were further analyzed for the different distances among cells (range 1-25 $\mu \mathrm{m}$ ), displaying no negative correlation between cell-cell contacts and distance between cells for all treatments (Figure 4c). Moreover, HO-1 silencing also reverted the augmented filopodia density after hemin exposure (Figure 4d).

Presence of $\mathrm{HO}-1$ at the leading edge protrusions of cells was also assayed by immunofluorescence. $\mathrm{HO}-1$ induction in PC3 showed increased expression of this protein in the cytoplasm and nuclei of cells but did not reveal localization at cell filopodia (Supplementary Figure S2).

Metastatic cells rely on invadopodia to degrade and invade $\mathrm{ECM}^{5}$ To rule out that these increased protrusions were invadopodia structures, we stained cells for metalloproteinase 9 (MMP9), a metalloproteinase highly implicated in tumor invasion. No staining for MMP9 was detected at these protrusions (Supplementary Figure S3).

Microtubule mechanics in PC3 cells treated or not with hemin was also evaluated by confocal microscopy (Supplementary Figure S4). The persistence length ( $p L)$ of microtubules was measured in PC3 cells. A Fourier analysis was performed and the ensemble variance of Fourier amplitudes was calculated. Both, cells treated or not with hemin, exhibited a similar thermal-like $q$-dependence: $\left(1 / L p^{\star}\right)^{\star}(1 / q)^{2}$ with no variation of $L p$ (Supplementary Figure S4).

Considering that PC3 cells present an osteolytic bone metastatic behavior, we extended our observations to $\mathrm{C} 4-2 \mathrm{~B}$ cells, another $\mathrm{PCa}$ cell line with osteoblastic metastatic behavior. Imaging of $\mathrm{C} 4-2 \mathrm{~B}$ cells also revealed an increased network of filopodia from neighboring cells and an augmented number of protrusions per cell by forced expression of $\mathrm{HO}-1$ (Supplementary Figure S5). HO-1 depletion could revert the increased contact density under hemin exposure (Supplementary Figure S5), however, no significant difference was observed in the filopodia density, probably due to a reduced efficiency of the siHO compared with the effect observed in PC3 cells. These results show that $\mathrm{HO}-1$ induction in $\mathrm{PCa}$ cells favors a more adhesive phenotype regardless the bone metastatic behavior.
Analysis of $\mathrm{PCa}$ cellular protrusions in the presence of CM from co-culture systems (PC3 and MC3T3). As bone is the most common and frequent site of $\mathrm{PCa}$ progression and the bioinformatics analysis has shown important dysregulation of cytoskeletal proteins in the metastatic stage of $\mathrm{PCa}$, we next assessed the effect of conditioned media (CM) obtained from transwell co-culture systems (PC3 and MC3T3), on tumoral cell protrusions. MC3T3 is an osteoblast precursor cell line derived from mouse calvaria. ${ }^{16}$

PC3 cells pre-treated or not with hemin were co-cultured with or without MC3T3 cells. CM from the different experimental protocols was then added to PC3 cells (Figure 5a). Cell contact density and number of protrusions were evaluated by confocal microscopy (Figure 5b). Regarding contacts between cells, no correlation was observed for contact density and cellcell distance for all conditions (Figure $5 \mathrm{c}$ ). $\mathrm{CM}$ from co-culture systems reduced the number of contacts among $\mathrm{PCa}$ cells (Figure 5d; $\mathrm{PC} 3+\mathrm{CM} 2$ versus $\mathrm{PC} 3+\mathrm{CM} 1$ ) and hemin pretreatment prevented this fall (Figure $5 \mathrm{~d}$; $\mathrm{PC} 3+\mathrm{CM} 4$ versus $\mathrm{PC} 3+\mathrm{CM} 2)$. Intriguingly, the $\mathrm{CM}$ from co-culture systems impacted negatively on the membrane filopodia density of PC3 cells (Figure 5d; PC3+CM2 versus PC3+CM1). These effects were prevented by hemin pre-treatment (Figure $5 \mathrm{~d}$; $\mathrm{PC} 3+\mathrm{CM} 4$ versus $\mathrm{PC} 3+\mathrm{CM} 2)$. These findings suggest that induced $\mathrm{HO}-1$ expression in $\mathrm{PC} 3$ cells alters the soluble factors released to the $\mathrm{CM}$ in the co-culture systems, in turn affecting cell filopodia and zippering. A more adhesive phenotype of tumoral cells potentially prevent them from extravasation and invasion of other homing organs.

Analysis of RNAseq data on PCa cells overexpressing HO-1 pharmacologically or genetically. Given the sound cellular evidence of $\mathrm{HO}-1$ modulation on $\mathrm{PCa}$ cell adhesiveness and protrusive forces, we carried out an RNA-Seq analysis to compare gene expression profiles between $\mathrm{PCa}$ cells overexpressing $\mathrm{HO}-1$ pharmacologically (hemin treatment) or genetically (transfected with pcDNA3HO-1) and their respective controls (Figure 6a). For both comparisons: $\mathrm{PC} 3 \mathrm{HO}-1$ versus PC3pcDNA3 (empty vector) and PC3 hemin versus PC3 control we obtained differential subsets of upregulated $(\geqslant 2, \geqslant 3, \geqslant 5, \geqslant 8$-fold change cut-off) and downregulated ( $\leqslant-2, \leqslant-3, \leqslant-5, \leqslant-8$-fold change cut-off) genes (Figure $6 b$ ). We screened for overlapping up or downregulated genes for both comparisons (on same threshold subsets). Results show 92 downregulated $(\leqslant-2$, $P<0.05)$ and 118 upregulated $(\geqslant 2, P<0.05)$ overlapping genes (Figure 6b). We selected these overlapping gene data sets and performed GO analysis identifying pathways in which these differentially expressed genes are involved and enriched in subcategories (Figure 6c). We performed a second heat map depicting the top 37 significantly regulated genes within these cytoskeletal-related categories (Figure 6d), highlighting key markers related to cell adhesion (blue) and cell-cell communication (light blue). We also built a transcriptomic interaction network, between the GO overlapping twofold upregulated gene categories (red) and twofold downregulated gene categories (blue) showing interconnectivity either by reported genetic interactions, common pathways, or physical interactions (Figure 6e). 
a

\begin{tabular}{|c|c|c|c|c|c|c|c|c|}
\hline$N^{\circ}$ & Study & Plataform & $\begin{array}{l}\text { Measured } \\
\text { genes }\end{array}$ & Reporter & $\mathbf{N}$ & P-value & $\begin{array}{l}\text { Fold } \\
\text { change }\end{array}$ & $\begin{array}{c}\text { GEO } \\
\text { accession }\end{array}$ \\
\hline 1 & Arredouani Prostate & $\begin{array}{l}\text { HG U133 Plus } 2.0 \text { Array } \\
\text { Agilent Human Genome }\end{array}$ & 19,574 & 54,675 & 21 & 0.05 & 1.5 & GSE55945 \\
\hline 2 & Grasso Prostate & $44 \mathrm{~K}$ & 19,189 & 41,000 & 122 & 0.05 & 1.5 & GSE35988 \\
\hline 3 & Holzbeierlein Prostate & HG U95A-Av2 Array & 8,603 & 12,651 & 54 & 0.05 & 1.5 & $N / A^{*}$ \\
\hline 4 & Lapointe Prostate & Undefined plataform & 10,166 & 19,116 & 112 & 0.05 & 1.5 & GSE3933 \\
\hline 5 & LaTulippe Prostate & HG U95A-Av2 Array & 8,603 & 12,651 & 35 & 0.05 & 1.5 & GSE68882 \\
\hline 6 & Liu Prostate & HG U133A Array & 12,624 & 22,283 & 57 & 0.05 & 1.5 & GPL1261 \\
\hline 7 & Luo Prostate & $\begin{array}{l}\text { Hu35k (A-D) and } \\
\text { HG U95A-Av2 array }\end{array}$ & 15,302 & 47,060 & 30 & 0.05 & 1.5 & GSE68545 \\
\hline 8 & Magee Prostate & HumanGeneFL Array & 5,338 & 7,133 & 15 & 0.05 & 1.5 & N/A \\
\hline 9 & Singh Prostate & HG U95A-Av2 Array & 8,603 & 12,651 & 102 & 0.05 & 1.5 & GSE68907 \\
\hline 10 & Taylor Prostate & Undefined plataform & 22,238 & 43,419 & 150 & 0.05 & 1.5 & GSE21032 \\
\hline 11 & Tomlins Prostate & Undefined plataform & 10,656 & 19,928 & 53 & 0.05 & 1.5 & GSE6099 \\
\hline 12 & Vanaja Prostate & HG U133(A-B) Array & 17,779 & 44,928 & 40 & 0.05 & 1.5 & N/A \\
\hline 13 & Varambally Prostate & HG U133 Plus 2.0 Array & 19,574 & 54,675 & 19 & 0.05 & 1.5 & GSE3325 \\
\hline 14 & Wallace Prostate & HG U133 2.0 Array & 12,603 & 22,283 & 89 & 0.05 & 1.5 & GSE6956 \\
\hline 15 & Welsh Prostate & HG U95A-Av2 Array & 8,603 & 12,651 & 34 & 0.05 & 1.5 & GPL96 \\
\hline 16 & Yu Prostate & HG U95A-Av2 Array & 8,603 & 12,651 & 112 & 0.05 & 1.5 & GSE68555 \\
\hline
\end{tabular}

b

\begin{tabular}{|c|c|c|c|}
\hline GENE & $\begin{array}{l}\text { MEDIAN } \\
\text { RANK }\end{array}$ & P-VALUE & $+\square \square \square[$ \\
\hline CBX1 & 2604.0 & 0.019 & पनायन \\
\hline LASP1 & 2112.0 & 0.009 & प्यात्र \\
\hline RPA1 & 2093.0 & $771 \mathrm{E}-4$ & बनियगजनाम \\
\hline MX1 & 1542.0 & 0.031 & गाशगयनान \\
\hline EEF1A1 & 1410.5 & 0.016 & तनलम \\
\hline NAP1L1 & 1198.0 & 0.021 & 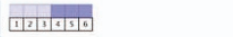 \\
\hline RAl14 & 1673 & 0.027 & 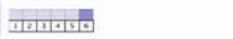 \\
\hline TES & 896.0 & 0.042 & प्राशान्य \\
\hline STAT3 & 885.0 & 0.002 & 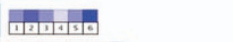 \\
\hline GSN & 586.5 & 0.005 & 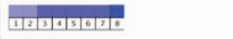 \\
\hline MKLN1 & 365 & 0.038 & तन \\
\hline HSPB1 & 182.5 & $1.064 \mathrm{E}-4$ & घनानांक \\
\hline ANXA2 & 133 & 0.003 & 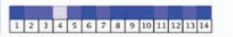 \\
\hline
\end{tabular}

c

\begin{tabular}{|c|c|c|c|c|}
\hline STUDY ABBREVIATION & STUDY NAME & $\begin{array}{l}\text { ALTEFED } \\
\text { CASES }\end{array}$ & $\begin{array}{l}\text { ALTEFED } \\
\text { CASES }\end{array}$ & N SAMPLES \\
\hline $\begin{array}{c}\text { NEPC } \\
\text { (Trento/Cornell/Broad 2016) }\end{array}$ & $\begin{array}{l}\text { Neuroendocrine Prostate Cancer } \\
\text { (Trento/Cornell/Broad 2016) } \\
\text { Prostate Adenocarcinoma }\end{array}$ & 56 & $52.3 \%$ & 114 \\
\hline Prostate (FHCRC) & $\begin{array}{l}\text { (Nelson Lab, Fred Hutchinson CRC) } \\
\text { Prostate Adenocarcinoma, Metastatic }\end{array}$ & 61 & $40.9 \%$ & 176 \\
\hline Prostate (MICH) & $\begin{array}{l}\text { (Michigan, Nature 2012) } \\
\text { Prostate Adenocarcinoma }\end{array}$ & 22 & $36.1 \%$ & 61 \\
\hline Prostate (TCGA 2015) & $\begin{array}{l}\text { (TCGA, Cell 2015) } \\
\text { Metastatic Prostate Cancer, SU2C/PCF Dream }\end{array}$ & 115 & $34.5 \%$ & 333 \\
\hline Prostate (SU2C) & $\begin{array}{l}\text { Team (Robinson et al., Cell 2015) } \\
\text { Prostate Adenocarcinoma }\end{array}$ & 34 & $22.7 \%$ & 150 \\
\hline $\begin{array}{l}\text { Prostate (MSKCC 2010) } \\
\text { Prostate }\end{array}$ & $\begin{array}{l}\text { (MSKCC, Cancer Cell 2010) } \\
\text { Prostate Adenocarcinoma }\end{array}$ & 10 & $9.7 \%$ & 216 \\
\hline (Broad/Cornell 2012) & $\begin{array}{l}\text { (Broad/Cornell, Nat Genet 2012) } \\
\text { Prostate Adenocarcinoma CNA study }\end{array}$ & 4 & $3.7 \%$ & 112 \\
\hline Prostate (MSKCC 2014) & (MSKCC, PNAS 2014) & 1 & $1 \%$ & 104 \\
\hline
\end{tabular}

\begin{tabular}{|c|c|c|c|}
\hline GENE & $\begin{array}{l}\text { MEDIAN } \\
\text { RANK }\end{array}$ & P-VALUE & 느 \% \\
\hline TMOD3 & 2035.0 & 0.016 & 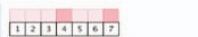 \\
\hline THRAP3 & 1898.0 & 0.002 & $1 / 2 / 3 / 45$ \\
\hline HMGA1 & 1643.0 & 0.019 & प्राp \\
\hline NAP1L1 & 1590.0 & 0.040 & पनल/4 \\
\hline TRIM28 & 1546.0 & 0.003 & 42 \\
\hline AHCTF1 & 1383.5 & 0.005 & मिगय \\
\hline SPAST & 1352.0 & 0.006 & 1213 \\
\hline TOP1 & 1082.0 & 0.003 & $\sqrt{12 / 3 / 4 / 567}$ \\
\hline СвХ3 & 825.0 & $421 \mathrm{E}-4$ & 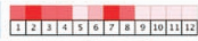 \\
\hline RCC1 & 487.0 & 0.012 & 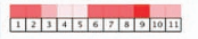 \\
\hline NPM1 & 117.5 & 7.33E-5 & (12) 1906 \\
\hline
\end{tabular}

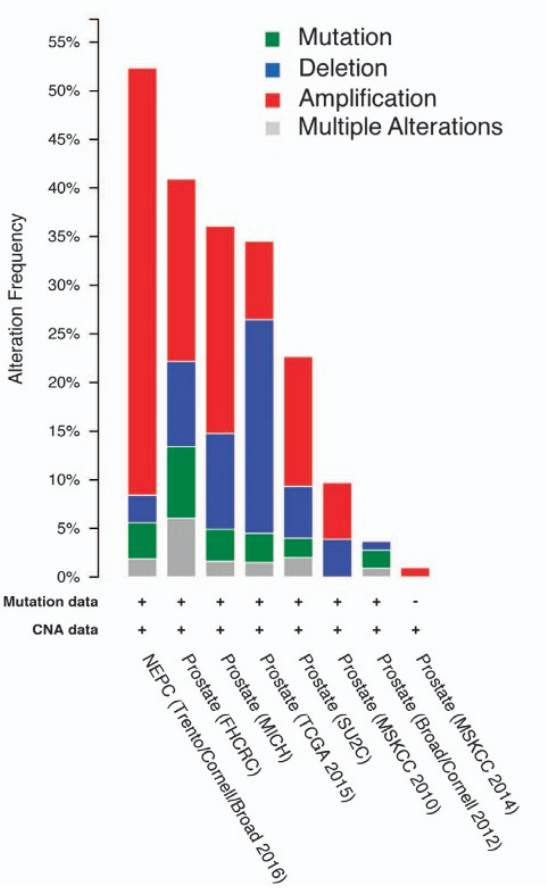


Integrated analysis of $\mathrm{HO}-1$ transcriptomic and proteomics data in PCa. We next integrated the transcriptomic overlapping data sets with the $\mathrm{HO}-1$ interactome for a combined holistic view of $\mathrm{HO}-1$ molecular mechanisms implicated in the cytoskeletal remodeling in $\mathrm{PCa}$. Figure $7 \mathrm{a}$ shows the interaction network between the cytoskeletal GO categories of the proteome with the overlapping twofold upregulated gene categories (red) and twofold downregulated gene categories (blue) of the trascriptome.

Filtering by physical interconnectivity among these data sets, four main molecular pathways were depicted: ANXA2 (Annexin 2) connected to PLAU (urokinase-type plasminogen activator precursor) signaling axis, HMGA1 (high mobility group AT-Hook) connecting to POU3F1, (POU class 3 homeobox 1) GSN (Gelsolin) with TNFRSF13C (tumor necrosis factor receptor superfamily member 13C) and TMOD3 (tropomodulin 3) and RAI1 (retinoic acid induced 1) with VWF (Von Willebrand factor) (Figure 7b, left panel). These pathways are intrinsically related to the cytoskeleton remodeling, MMP secretion, UPA pathways, Rho GTPases pathway and filopodia and lamillopodia regulation (Figure $7 \mathrm{~b}$, right panel). This network creates a molecular framework by which $\mathrm{HO}-1$ operates at the molecular level governing cell protrusive forces, migration, invasion and cell adhesiveness.

Interestingly, UPA/UPAR, through the activation of the plasminogen system, degrade ECM, and consequently drive tumor cell membrane protrusion and motility. ${ }^{17}$ The RNAseq profiling for both PCa cells overexpressing $\mathrm{HO}-1$ pharmacologically or genetically showed a direct regulation of the critical factors in the uPA/uPAR cascade, such as the downregulation of the axis activators: UPA/UPAR (PLAU/PLAUR) and tPA (PLAT), and the upregulation of the axis inhibitors: CPB2 (thrombin activator of fibrinolysis inhibitor), SERPINF2 (alpha 2 antiplasmin, and F12 (factor XIIA). Our data delineate a molecular axis by which $\mathrm{HO}-1$ potentially shuts down the acquisition of an invasive tumor cell phenotype, crucial for cancer metastasis.

\section{Discussion}

Different sets of genes, proteins and metabolites govern progression from a precursor lesion, to the localized disease and finally to the metastatic stage. Although several studies have profiled PCa tissues at the transcriptome level, less work has been done at the protein level that serve as the functional effectors of cancer progression.

Several studies have shown the anti-inflammatory effects of $\mathrm{HO}-1$ on diseases ${ }^{18-20}$ although the underlying mechanisms are still to be deciphered. Its role in cancer is still controversial. ${ }^{12,21-25}$ In $\mathrm{PCa}, \mathrm{HO}-1$ impairs cell proliferation, migration invasion, and angiogenesis in vitro and in vivo. ${ }^{14}$ The confirmation of its association with the augmented adhesive capabilities of cells and the induction and relocation of E-cadherin and $\beta$-catenin to the cell membrane, ${ }^{13}$ led us to hypothesize its potential implications in cell-cell adhesion zippering and in the regulation of the actin dynamics at the leading edge of cells.

For this purpose, we built for the first time the $\mathrm{HO}-1$ interactome in PCa cells, showcasing 56 molecular partners, including cytoskeletal proteins with roles in cell structure, physiological processes, cell signaling and regulation of the actin stress fiber dynamics. Pharmacological HO-1 induction altered migratory patterns and PCa cell trajectories toward a less motile phenotype.

Cell-cell adhesion is essential for the development, differentiation and maintenance of tissues. Epithelial cells require cell-cell adhesion zippering to promote barrier function. ${ }^{8}$ This barrier is critical in the maintenance of cellular homeostasis. Its loss provides a considerable advantage for carcinoma progression, as disruption of cell-cell interactions and detachment from the ECM are required for proliferation, aberrant signaling, epithelial-to-mesenchymal transition, invasion and metastasis. ${ }^{7}$ These events are facilitated by the actin cytoskeleton and tubulin at microtubules. ${ }^{26}$ Actin is the key component of several cellular structures, including filopodia, lamellipodia and stress fibers. Actin bundling contributes to epithelial cell polarity by preserving cell-cell junctions and microvilli. ${ }^{26}$ In the metastatic disorder, actin and the cytoskeletal proteins serve as mechanosensors between the cell and the microenvironment. ${ }^{26}$ There is no general rule whether actin bundling promotes or inhibits cancer metastasis. ${ }^{26}$ Mechanical stiffness has long been positively associated with invasion and metastasis. ${ }^{27}$ However, Swaminathan et al. ${ }^{28}$ reported that cancer cells with the highest migratory and invasive potential are significantly less stiff than cells with the lowest migratory and invasive potential. It is clear that transformed cells display corrupt actin bundling, turning the cytoskeletal compartment into a key target to understand tumor progression and develop appropriate therapies.

By confocal microscopy, we quantified and compared actin filaments at the leading edge level in PCa cells. HO-1 induced the remodeling of the actin filament architecture at filopodia, yielding a more adhesive and less invasive phenotype. The fact that $\mathrm{HO}-1$ silencing could revert the augmented contact density observed under $\mathrm{HO}-1$ forced expression, showcases a direct effect on cellular actin-based protrusions. However, $\mathrm{HO}-1$ displays negative staining at cell filopodia, pointing to a regulatory role of this protein on the actin filaments, rather than a direct interaction between these proteins.

Figure 2 Meta-analysis of multiple microarray data sets for cytoskeletal HO-1-interacting proteins. (a) Expression microarray studies selected from the Oncomine platform (http://www. oncomine. org) comparing prostate adenocarcinoma versus normal prostate. (b) Summary table showing the gene name, median gene rank and corresponding $P$-value for the HO-1 cytoskeleton-related interacting proteins. The median rank for a gene is the rank for the gene across each of the analyses. The $P$-value for a gene is the $P$ value for the median ranked analysis. Right panel shows a heat map indicating the level of expression for each gene in each study selected (blue: least expressed, red: most expressed). Each square represents the number of studies that met our eligibility criteria and thresholds. Colors are z-score normalized to depict relative values within a row. (c) Studies selected from the $c B i o P o r t a l$ for Cancer Genomics platform (www.cbioportal.org) summarizing the total number of cases with alterations for each study (left panel). The graph (right panel) shows the percentage of altered cases ( $y$ axis) and the alteration type observed whether it corresponds to mutations or copy number alterations (CNA) for each study ( $x$ axis). Mutations are shown in green, deletions in blue, amplifications in red and multiple alterations in gray, for the cytoskeleton-related proteins mentioned in (b) across the seven data sets selected comparing prostate tumor tissue versus normal prostate 
Men with PCa display characteristically osteoblastic bone metastases, which are the main cause of morbidity and mortality of the disease. ${ }^{29}$ A previous report has shown $\mathrm{HO}-1$ potentiality in modifying the bone microenvironment and subsequent $\mathrm{PCa}$ metastases. ${ }^{30}$ To explore the contribution of $\mathrm{HO}-1$ in the interaction between $\mathrm{PCa}$ cells and osteoblasts, a

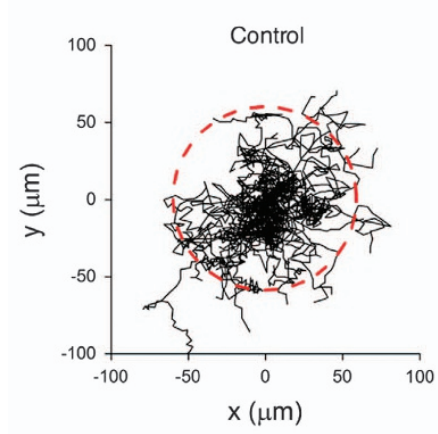

c
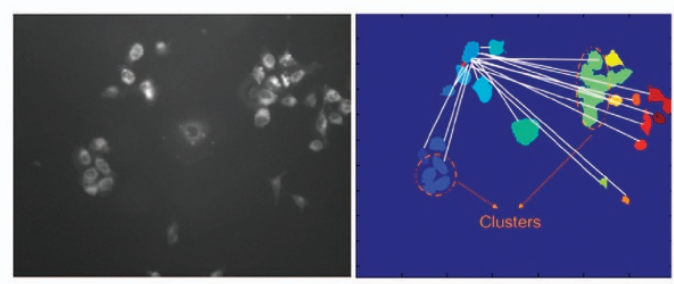

e

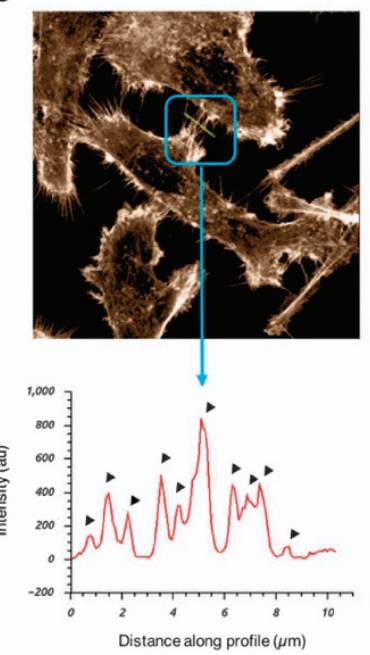

g
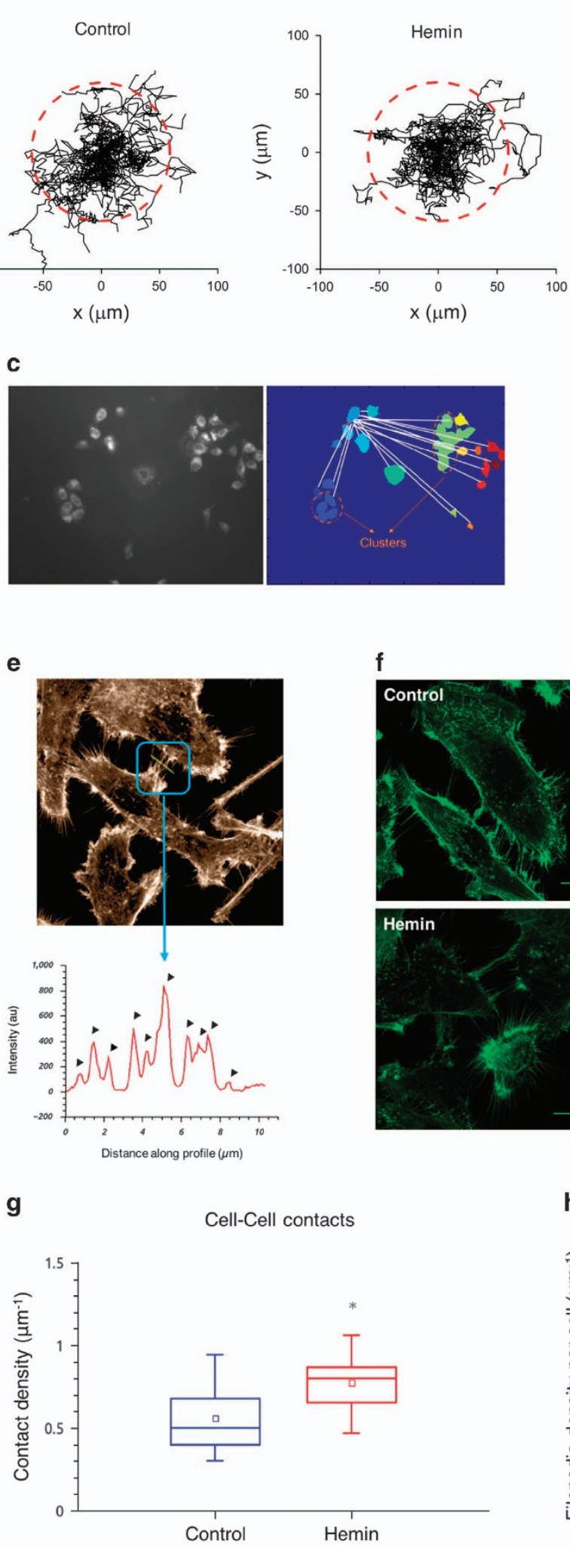

b

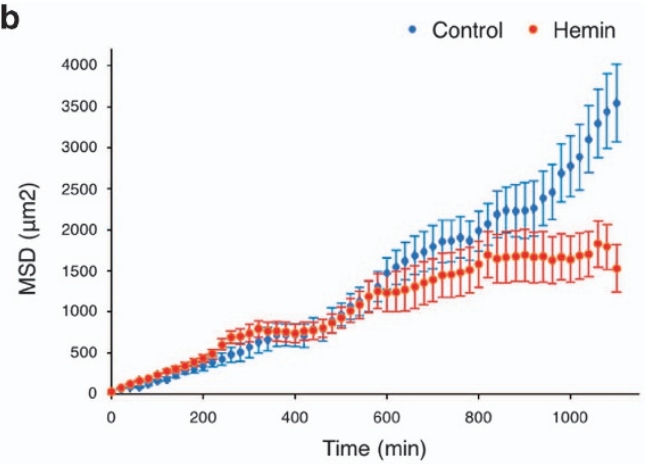

d

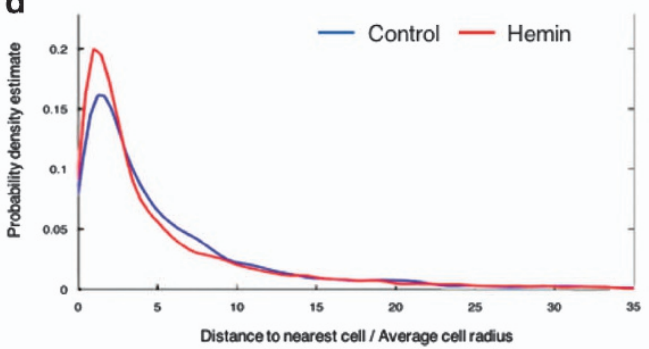

f
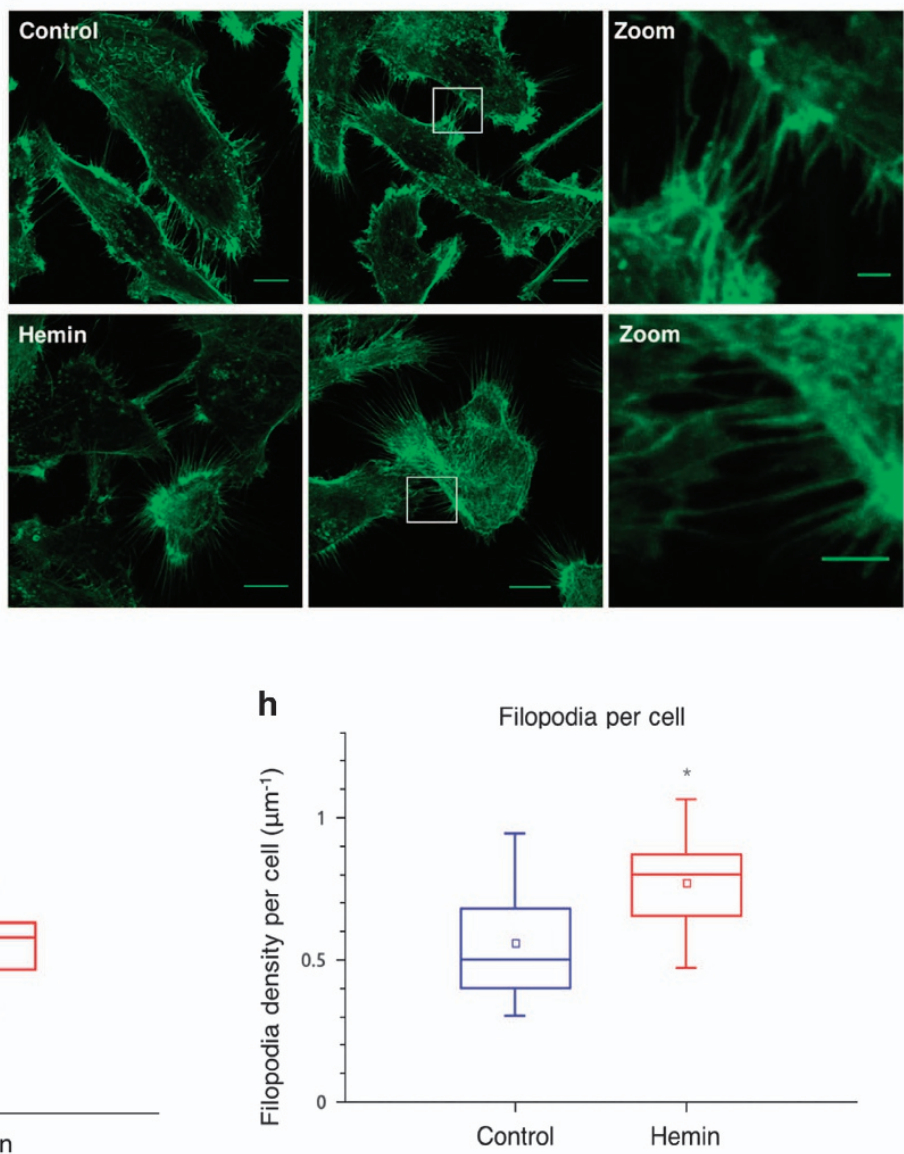
we used co-culture systems (PC3 and MC3T3). PCa cells exposed to CM from these co-culture systems, displayed reduced membrane filopodia density and contact among cells, effects reverted by hemin pre-treatment. Retraction of cell protrusions could be a sign of rounding up of cells and may be one of the characteristic features of cell detachment. Our results suggest that $\mathrm{HO}-1$ prevents $\mathrm{PCa}$ cells from extravasation and invasion to other homing organs.

To point out the significance of the cytoskeletal HO-1interacting proteins in prostate carcinogenesis, we searched the public cancer microarray database Oncomine and cBioportal. Essential transcriptional features for lethal castration-resistant $\mathrm{PCa}$ were discovered using metaanalysis of transcriptomic data. ${ }^{31,32}$ Interestingly, TES and MKLN lie within the 1-9\% of the lowest expressed genes across this comparison. TES, a scaffold protein that participates in the reorganization of the actin cytoskeleton, ${ }^{33}$ is known for being a tumor suppressor in PCa. ${ }^{34}$ MKLN1, a nucleocytoplasmic mediator of cellular morphology and adhesiveness, ${ }^{35}$ exhibits similar sub-cellular dynamics to $\mathrm{HO}-1$, relocating from the cell membrane toward the cell nuclei under hemin treatment. ${ }^{13}$

Multi 'omics' technologies reveal new aspects of the mechanistic strategy that $\mathrm{HO}-1$ uses to alter protrusive forces and adhesiveness of tumor cells, which may not otherwise be deduced from a single-omics approach. The RNA profiling evidenced significant alterations of key markers related to cell adhesion and cell-cell communication under $\mathrm{HO}-1$ induction. Strikingly, when integrating the transcriptome under $\mathrm{HO}-1$ modulation with the HO-1 interactome, a framework of four main molecular pathways arose as the foundation for the regulation of the tumor cell protrusive forces: ANXA2/HMGA1/ POU3F1; NFRSF13/GSN; TMOD3/RAI14/VWF; PLAT/PLAU. We provide a schematic representation of the different cytoskeletal-associated targets modulated by $\mathrm{HO}-1$ overexpression, which is supported by the results presented here and our previous data. ${ }^{13,14}$ Of note, downregulation of UPA/ uPAR directly impacts on Rho GTPases pathways through the alpha V-Beta 3 integrin receptor, which in turn affect filopodia formation. HO-1 binds Gelsolin, STAT3 and HSPB1, potentially supporting its implication in filopodia regulation and gives ground to $\mathrm{HO}-1$ involvement at the molecular level in the modulation of the cytoskeleton pathways.

\section{Materials and Methods}

Cell culture, treatments, reagents and antibodies. PC3 cells were obtained from the American Type Culture Collection (Manassas, VA, USA) and were routinely cultured in RPMI 1640 (Invitrogen, Grand Island, NY, USA) supplemented with $10 \%$ fetal bovine serum (FBS). PC3 cells were transiently transfected with HO-1FLAG plasmid or FLAG empty vector.

Hemin was obtained from Sigma-Aldrich (Glasgow, UK). For treatments, cells were incubated $24 \mathrm{~h}$ in RPMI media containing 10\% FBS and then were exposed to hemin $(80 \mu \mathrm{M}, 24 \mathrm{~h})$.

For $\mathrm{H}_{2} \mathrm{O}_{2}$ treatment, cells were exposed to $\mathrm{H}_{2} \mathrm{O}_{2}(200 \mu \mathrm{M}, 0.5 \mathrm{~h})$.

Polyclonal and monoclonal anti-HO-1 antibodies were from Stressgen Biotechnologies Corp. (San Diego, CA, USA). Anti- $\beta$-actin antibody was purchased from SigmaAldrich (UK). The rhodamine-phalloidin was purchased from Life Technologies (Thermo Fisher Scientific Inc., Eugene, OR, USA). Anti-mouse and anti-rabbit secondary antibodies conjugated with HRP were from Amersham Ltd (Freiberg, Germany). Secondary antibodies conjugated with Alexa Fluor 488 or Alexa Fluor 555 were from Molecular Probes, Invitrogen (Grand Island, NY, USA).

p3xFLAG-CMV-10-HO-1 cloning. p3xFLAG-CMV-10-HO-1 vector was generated by cloning the CDNA sequence encoding the human HO-1 (HMOX1) into the restriction sites $E c o R I$ and $B a m H I$ of the mammalian expression vector p3xFLAG-CMV-10 (Sigma-Aldrich, UK). This strategy results in the fusion of the Flag tag peptide at the $\mathrm{C}$-terminus of the HMOX1. The sequences of the primers used were as follow: forward - 5'-GCAGAATTCAGAGCGTCCGCAACCC-3'; reverse - 5' - GCCGGATCCGCATTCACATGGCATAAAGC-3'.

FLAG immunoprecipitation strategy. PC3 cells were transfected either with FLAGHO-1 plasmid or the empty vector as a control. Forty-eight hours after transfection, proteins were extracted using a buffer with a low $\mathrm{NaCl}$ concentration (20 mM Tris, $150 \mathrm{mM} \mathrm{NaCl}, 5 \mathrm{mM} \mathrm{MgCl}_{2}, 0.5 \% \mathrm{NP} 40, \mathrm{pH} 7,5$ ) not to disrupt the protein-protein interactions. Protein extracts were incubated with Flag Magnetic Beads (purchased from Sigma-Aldrich, UK) for $2 \mathrm{~h}$ at $4^{\circ} \mathrm{C}$. After removing the proteins that did not interact with the FLAG construction, the proteins complexes formed were incubated with 3XFLAG peptide $(100 \mu \mathrm{g} / \mu \mathrm{l})$ for $2 \mathrm{~h}$ at $4{ }^{\circ} \mathrm{C}$. Flag peptide competes with the proteins complexes bound to the magnetic beads and, as a consequence, the FLAGHO-1-interacting proteins remain in the supernatant.

Separation of peptides and mass spectrometry. Recombinant FLAGHO-1 protein complexes were reduced (200 mM DTT), alkylated (200 mM iodoacetamide) and digested with trypsin in-solution overnight, using an estimated $1: 30$ enzyme to substrate ratio. The peptides were desalted and concentrated in a C18 resin (Zip-Tips, Waters Technologies Corporation, Milford, MA, USA) before analysis by LC-ESI MSMS at the Center for Metabolomics and Mass Spectrometry (The Scripps Research Institute, La Jolla, CA, USA). Peptides were separated by reverse-phase chromatography before mass spectrometry analysis using the following method: nanoelectrospray capillary column tips were made in-house by using a P-100 laser puller (Sutter Instruments, Novato, CA, USA). The columns were packed with Zorbax SB-C $\mathrm{C}_{18}$ stationary phase (Agilent Technologies, Santa Clara, CA, USA) purchased in bulk (5-mm particles, with a 15-cm length and a

Figure 3 HO-1 overexpression impairs cellular trajectories in PCa cell lines and modulates the filopodia-like protrusions among neighboring PCa cells. Control and hemin $(80 \mu \mathrm{M}, 24 \mathrm{~h})$ treated PC3 cells were grown to confluence into a monolayer in $35 \mathrm{~mm}$ Petri dishes. A linear scratch wound was done along the culture plate and cell movement was monitored at a rate of 1 frame/20 min. (a) Representative cell trajectory plots obtained for control (left panel) or hemin-treated PC3 cells (right panel). Red dashed circles represent the mean area explored by PC3 cells after $1100 \mathrm{~min}$. (b) Trajectories were analyzed to obtain the MSD (mean square displacement) plot for control or hemin-treated cells. Data represent the average MSD \pm standard error over time, obtained from the analysis of 78 and 70 trajectories of control and hemin-treated cells, respectively. (c) PC3 cells treated with hemin $(80 \mu \mathrm{M}, 24 \mathrm{~h})$ or vehicle (control) were fixed and stained with C-Laurdan and images of large fields containing several cells were acquired using a 10x objective in an Olympus Wide Field Microscope (left panel). The images were analyzed with an algorithm designed to binnarize the image and assign a label to each set of connected pixels. In the right panel, each color represents a different cell or cell cluster (orange dotted circles). For every cell or cell cluster, the distances to all the other cells/cell clusters (white lines) and to the first neighboring cell (red line) were calculated. Distances were normalized to the average cell radii. (d) The distribution of the distances to the first neighbor cell was plotted for control and hemin-treated PC3 cells. The $x$ axis displays the cell-cell distances (using a representative cell radius as scale) and the $y$ axis represents the corresponding probability amplitude ( $N \geqslant 2200$ first neighbor distances for each condition). (e) PC3 cells treated with hemin or vehicle (control) were fixed and stained with rhodamine-phalloidin. Cells were imaged by confocal microscopy. The regions, in which cell filopodia contacted two neighboring cells, were divided into segments where the distance between the cells remained constant (blue frame). An intensity profile (yellow line) for each of these sectors was determined using a custom made algorithm to count contacts (Matlab). Arrow heads represent the intensity peaks considered as filopodia. (f) Two representative images for each group are shown (scale bars: $10 \mu \mathrm{m}$ ). White open boxes represent the zoomed images (scale bars: $2 \mu \mathrm{m}$ ). (g) Boxplot comparing the cell-cell contact density for the different experimental conditions. (h) The individual cell filopodia density was measured in hemin-treated and control cells ( $N \geqslant 91$ for each condition). *Significant difference, $P<0.001$ 
a
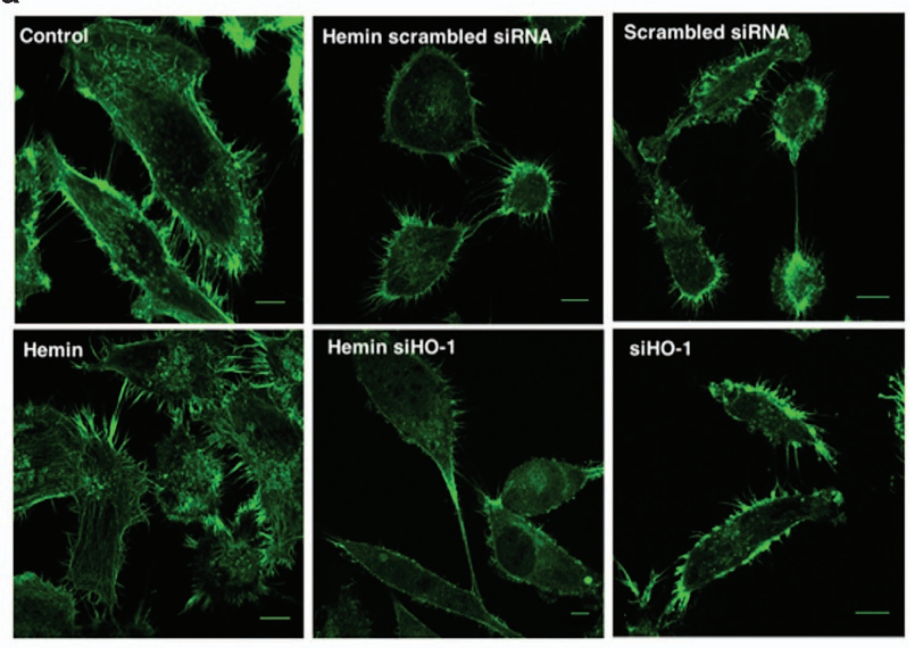

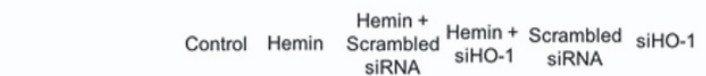

HO-1 (32KDa)

$\beta$-Actin (42KDa)

Fold induction
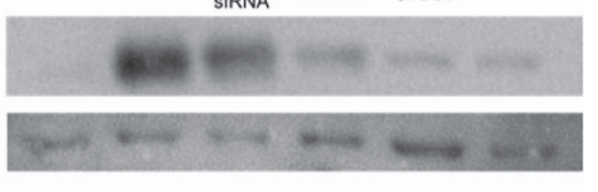

0.7 b

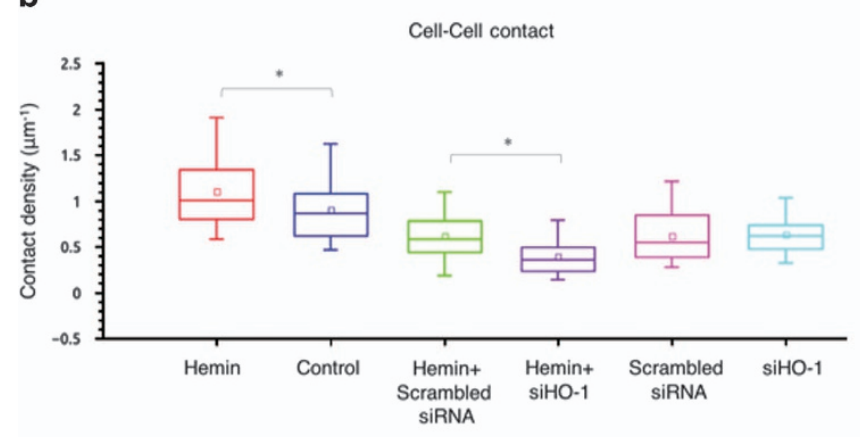

C

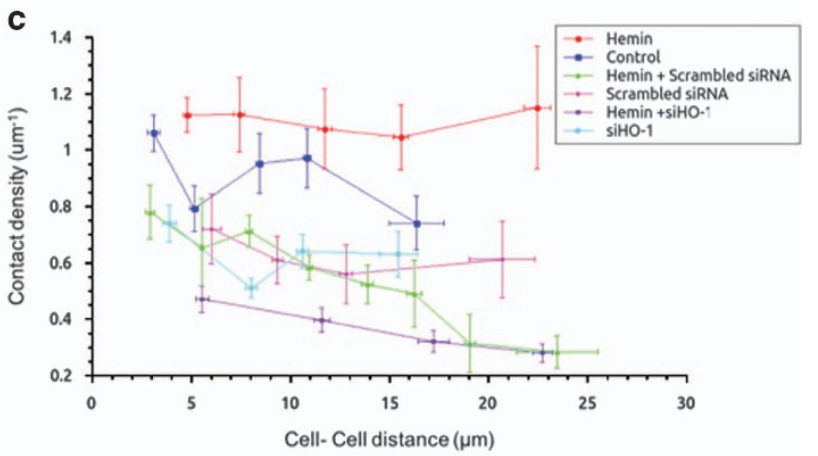

d Filopodia per cell

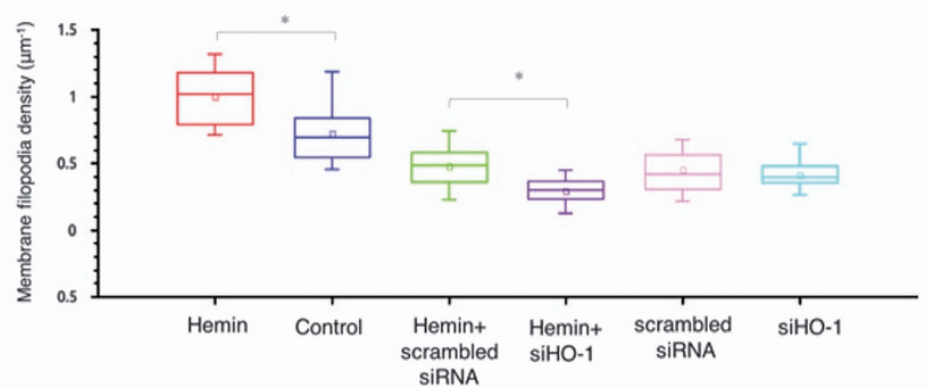

Figure 4 Filopodia-like protrusions under HO-1 modulation using siHO-1. (a) PC3 cells were treated with hemin ( $80 \mu \mathrm{M}, 24 \mathrm{~h}$ ) or vehicle (control) and transfected with a specific siRNA for HO-1 (siHO-1) or scrambled siRNA (control). Cells were fixed and stained with rhodamine-phalloidin and imaged by confocal microscopy. One representative image for each group is shown (left panel). Efficiency of siHO-1 was confirmed by western blot. $\beta$-Actin was used as loading control (right panel). (b) Cell-cell contact density versus cell-cell distance. The amount of contacts between cells was obtained and a linear density was determined by computing the number of contacts per unit perpendicular distance. Each data point represents the mean contact density within a bin determined by Freedman-Diaconis rule and error bars represent standard error of the mean ( $N \geqslant 33$ contact regions for each condition). *Significant difference, $P<0.001$. (c) Boxplot comparing the cell-cell contact density versus cell distance for the different experimental conditions. The regions, in which cell filopodia contacted two neighboring cells, were divided into segments where the distance between the cells remained constant. An intensity profile for each of these sectors was determined using a custom-made algorithm to count contacts (Matlab) $\left(N \geqslant 33\right.$ contact regions for each condition; $\left.{ }^{*} P<0.001\right)$. (d) Boxplot comparing filopodia density on single cells for the different experimental conditions. The density was measured by scanning an intensity profile around the cell perimeter and evaluating the amount of filopodia per unit length ( $N \geqslant 19$ cells for each condition; ${ }^{*}$ significant difference, $\left.P<0.001\right)$

75-mm inner diameter). The reverse-phase gradient separation was performed by using water and acetonitrile ( $0.1 \%$ formic acid) as the mobile phases. The gradient consisted of $5 \%$ acetonitrile for 10 min followed by a gradient to $8 \%$ acetonitrile for $5 \mathrm{~min}, 35 \%$ acetonitrile for $113 \mathrm{~min}, 55 \%$ acetonitrile for $12 \mathrm{~min}, 95 \%$ acetonitrile for $15 \mathrm{~min}$ and re-equilibrated with $5 \%$ acetonitrile for $15 \mathrm{~min}$.

Data-dependent MS/MS data were obtained with an LTQ linear ion trap mass spectrometer using a home-built nanoelectrospray source at $2 \mathrm{kV}$ at the tip. One MS spectrum was followed by $4 \mathrm{MS} / \mathrm{MS}$ scans on the most abundant ions after the application of a dynamic exclusion list. Tandem mass spectra were extracted by use of Xcalibur software (Thermo Scientific, Waltham, MA, USA). All MS/MS samples were analyzed by using Mascot (version 2.1.04; Matrix Science, London, UK) with H. Sapiens proteins contained in the NCBI protein database, assuming the digestion enzyme trypsin. Mascot was searched with a fragment ion mass tolerance of $0.80 \mathrm{Da}$ and a parent ion tolerance of $2.0 \mathrm{Da}$; identification was done at the $95 \%$ confidence level with a 


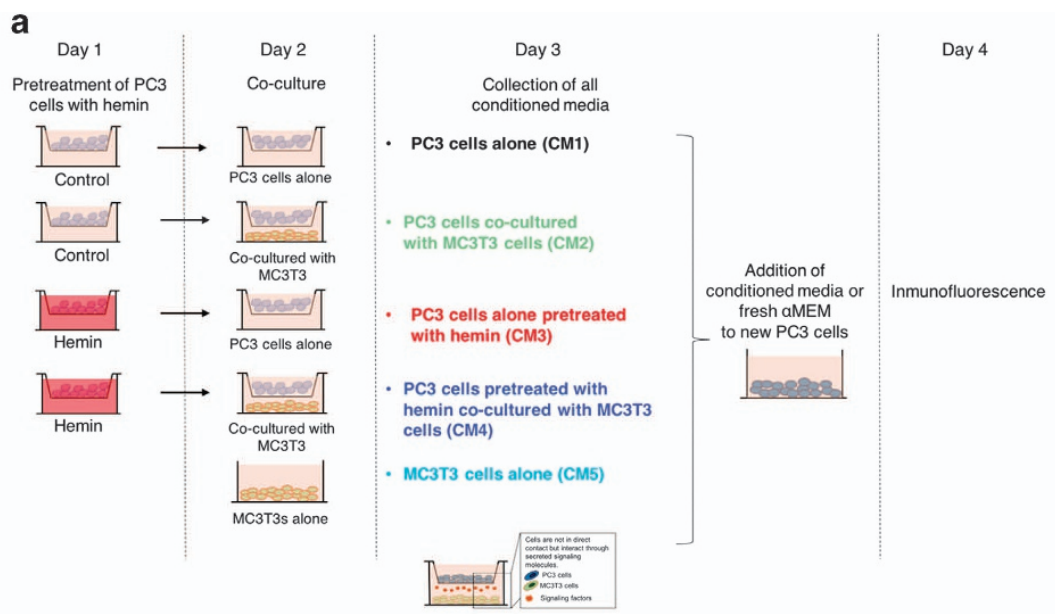

b
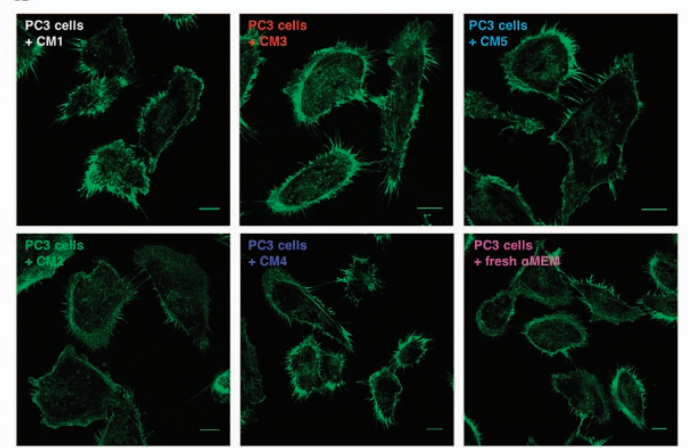

d

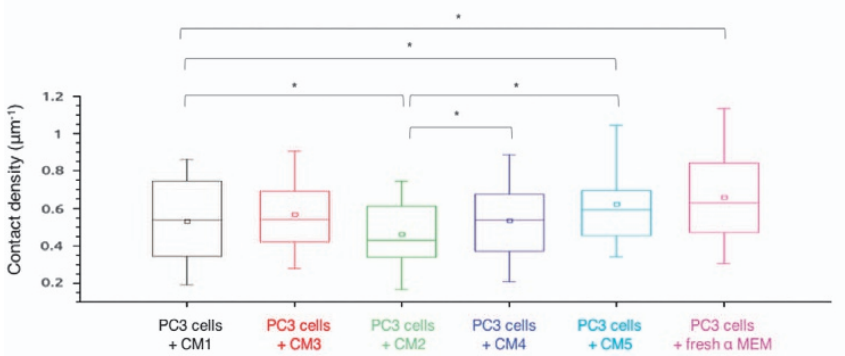

C

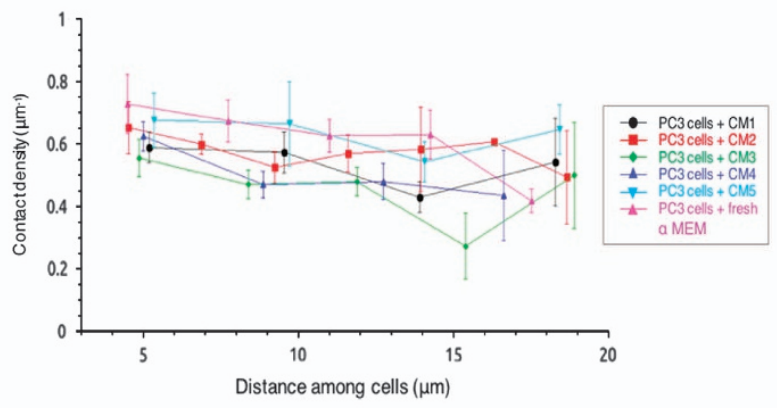

e

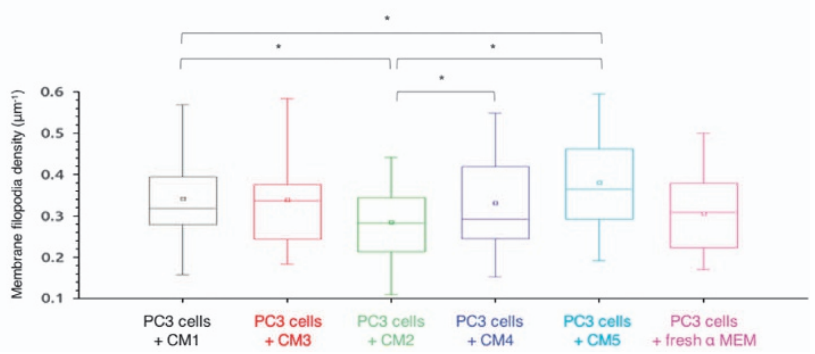

Figure 5 Analysis of cell contact density in PC3 cells cultured with CM from PC3-MC3T3 co-culture systems. PC3 cells were treated or not with hemin (80 $\mu \mathrm{M}, 24 \mathrm{~h}$ ) and then co-cultured with or without MC3T3 cells (24 h), in different compartments (insert membrane and well). CM from the different experimental conditions was then added to PC 3 cells for $24 \mathrm{~h}$. (a) Schematic representation of the experimental design. (b) Cells were fixed and stained with rhodamine-phalloidin and imaged by confocal microscopy to assess contact density and number of protrusions (scale bars: $10 \mu \mathrm{m}$ ). One representative image for each group is shown. (c) Cell-Cell contact density versus cell-cell distance for all treatments. For each area where two cells were in contact, the amount of contacts was obtained and a linear density was determined by computing the number of contacts per unit perpendicular distance. Each data point represents the mean contact density within a bin determined by Freedman-Diaconis rule and error bars are the standard error of the mean ( $N \geqslant 37$ contact regions per condition). (d) Boxplot comparing cell-cell contacts densities for the different experimental conditions. An intensity profile for each of these sectors was determined using a custom made algorithm to count contacts (Matlab) ( $N \geqslant 37$ contact regions per condition; $\left.{ }^{*} P<0.001\right)$. (e) Boxplot comparing the measured filopodia density per cell for the different experimental conditions. Error bars represent standard error $\left(N \geqslant 36\right.$ cells per condition; $\left.{ }^{*} P<0.001\right)$

calculated false-positive rate of $<1 \%$ as determined by using a reversed concatenated protein database. Peptide identifications were accepted if they could be established at a $>95.0 \%$ probability as specified by the Peptide Prophet algorithm. Protein identifications were accepted if they could be established at a $>99.0 \%$ probability and contained at least two identified peptides as specified by the Protein Prophet algorithm. ${ }^{36}$ Proteins that contained similar peptides and could not be differentiated based on MS/MS analysis alone were grouped to satisfy the principles of parsimony. To control for nonspecific binding, we compared FLAGHO-1 co-purifying proteins with those immunoprecipitated in cells transfected with a FLAG empty vector. Only differential FLAGHO-1 binding proteins compared with FLAG-binding proteins were considered further.

Bioinformatics data analysis. In all cases, the networks were performed using Cytoscape 3.2.1 software (Institute of Systems Biology, Seattle, USA). GO analysis was performed using the Database for Annotation, Visualization and Integrated Discovery (DAVID) v6.7. (Leidos Biomedical Research, Inc., Bethesda, MD, USA). 
Hemin pre-treatment of PCa cells and Co-culture system. An in vitro bicompartment culture system was used as a model of bone metastases from PCa as previously described slightly modified. Briefly cells were seeded (100 000 cells) in cell culture inserts $(0.4-\mathrm{mm}$ pore; Falcon/Becton Dickinson
Labware, Franklin Lakes, NJ, USA) and on day 1 they were treated with hemin ( $50 \mu \mathrm{M}$, Sigma-Aldrich, St. Louis, MO, USA), a potent inducer of HO-1 (PC3 Hem). Controls received fresh medium. The МСЗТЗ cells were also seeded on day 1 in tissue culture plates (100 000 cells per well). On day 2, the inserts containing the a
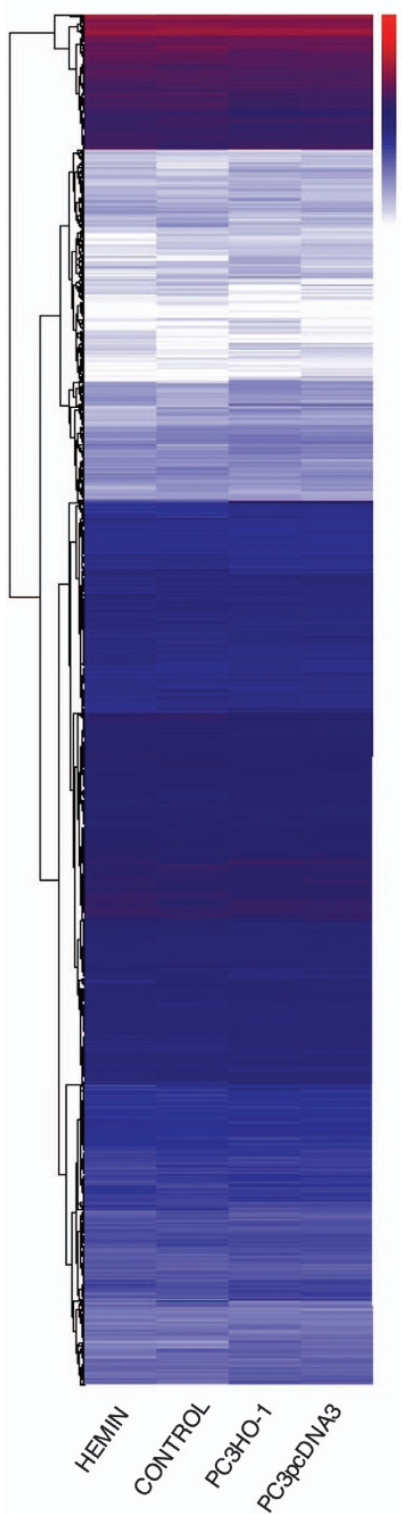

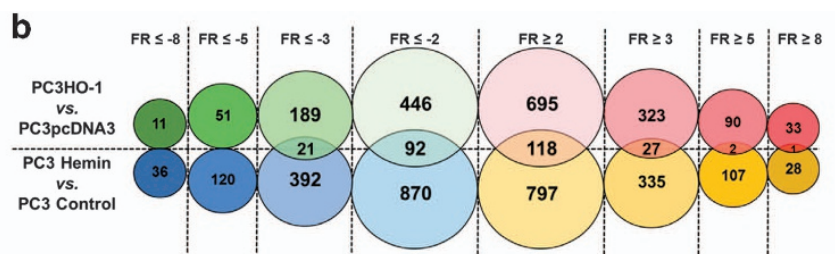

C
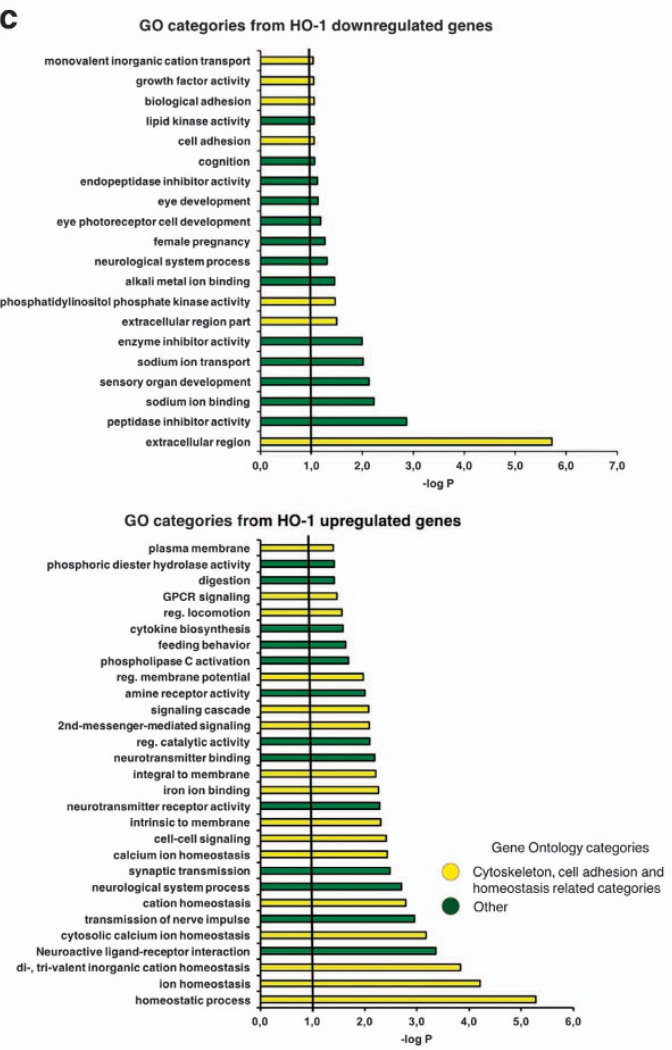

e

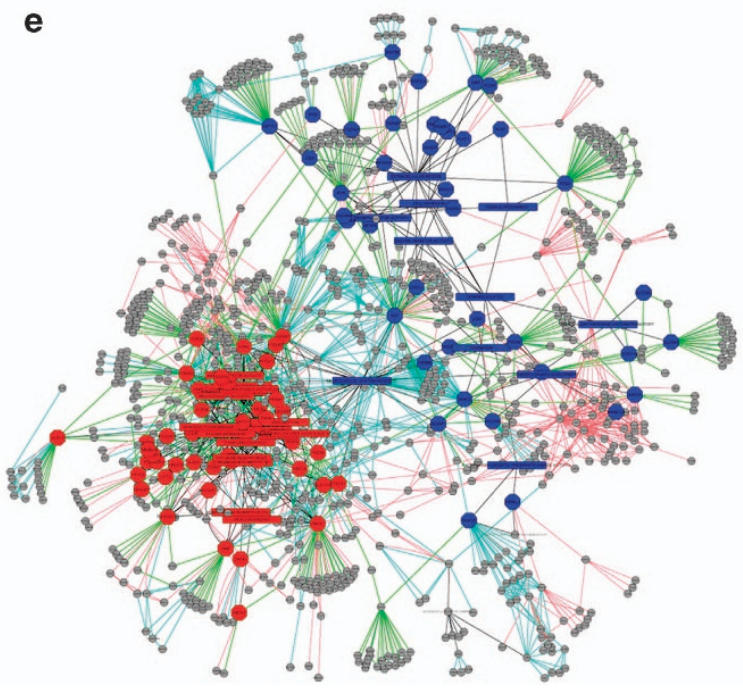

d

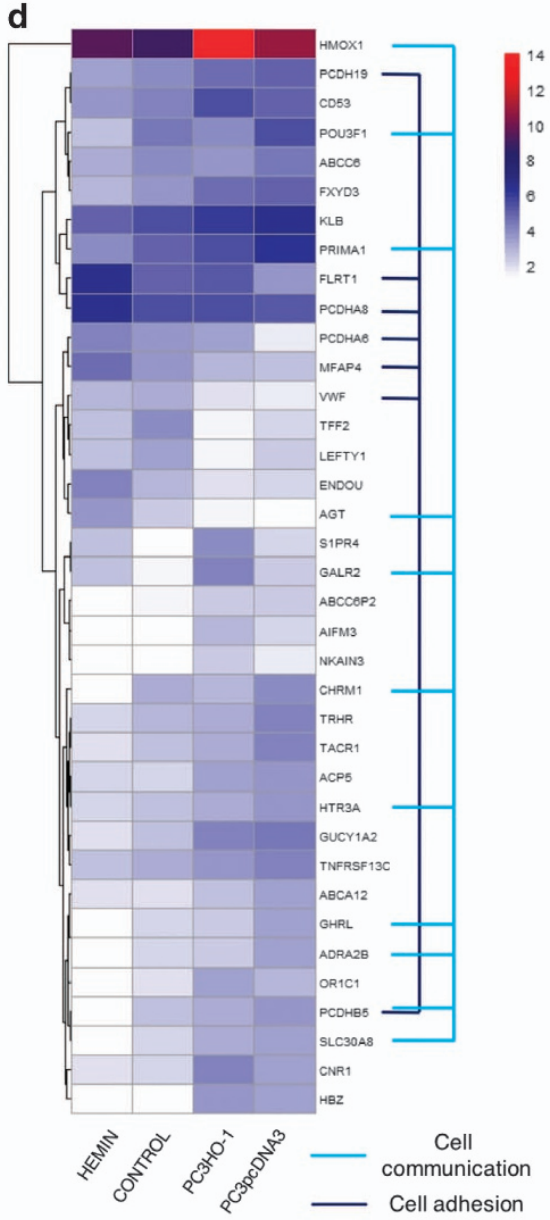


PC3 cells (pre-treated or not with hemin) were extensively washed with PBS. Then, the inserts were placed into tissue culture plates containing MC3T3 so that the two different cell types shared the culture medium but were not in physical contact. Co-culturing of PC3 cells with MC3T3 was performed with $\alpha$-MEM plus 2\% FBS for $24 \mathrm{~h}$. As control, each cell type (PC3 cells and MC3T3 cells) was grown alone. On day 3 , all $\mathrm{CM}$ were collected and used to incubate new PC3 cells for $24 \mathrm{~h}$ so as to perform immunofluorescence and filopodia analysis on day 4. Cultures were done in triplicate and each experiment was assayed three times.

siRNA transfection. siRNA anti-HO-1 (siHO-1) was used to knock down HO-1 expression and siRNA scramble was used as a negative control (ON-TARGETplus siRNA reagents, both purchased at Dharmacon, Pittsburgh, Pennsylvania, USA). The HO-1 siRNA is a pull of four targeted siRNA (HO-1 siRNA cat\# L-006372-00-05, scrambled siRNA cat\# D-001810-01-05). In all, $1 \times 10^{6}$ PC3 cells were grown in 12-well plates until $60 \%$ confluence and then transfected using Dharmacon (US) transfection reagent (Dharmacon) in medium supplemented with $10 \%$ FBS without antibodies. One day after transfection, the medium was replaced for complete medium including or not hemin $(80 \mu \mathrm{M}, 24 \mathrm{~h})$. Forty-eight hours after transfection, cells were fixed and stained for immunofluorescence experiments.

Bioinformatics inference on protein-protein interactions. Reference amino-acid sequences for EEF1A1, GSN, HSPB1, LASP1, MEGF10, MKLN1, MX1, NT5C2, RAl14, TES, TMOD3 (NP_001393.1, NP_001121135.2, NP_001531.1, NP_006139.1, NP_115822.1, NP_037387.2, NP_001171517.1, NP_001127845.1, NP_001138993.1, NP_056456.1, NP_055362.1, respectively) were annotated from RefSeq and a search for similar protein structures was achieved using Basic Alignment and Search Tool (BLAST) against the Protein Data Bank (PDB). The resulting structures were selected by assessment of the alignment coverage and score. Domain data were collected from the Protein Family database (PFAM). HO-1 interactor candidate protein domains were annotated and searches in the Interaction Protein Family (iPfam) database were performed, in order to get structural description of domain-domain interactions. The information gathered from these databases was used as input for the construction of an interaction network using Cytoscape 3.2.1 software.

\section{Bioinformatics analysis}

Information source and eligibility criteria (Oncomine): We searched the public cancer microarray database, Oncomine (715 data sets investigating and 68 tumor types), to identify expression microarray data sets that compared the expression of prostate adenocarcinoma versus prostate gland. In order to be included in our study, a data set was required to (1) be generated from human tumors, (2) compare prostate adenocarcinoma versus prostate gland, (3) have a $P$ value $<0.05$ and (4) have a fold change $>1.5$ and/or (5) have a gene rank between 1 and $10 \%$. It is worth mentioning that although the $P$-value criteria was strict for the data set selection, some genes were considered even if the fold change or the gene rank was $<1.5 \%$ or $>10 \%$, respectively, when the gene showed a significant over or under expression.

Search/study selection (Oncomine): We performed one search for each gene using the 'gene symbol' as the search term and obtained different number of studies for each gene. The selected studies were analyzed on the basis of normal gland versus prostate adenocarcinoma. Cited literature was reviewed to confirm that the analysis was as documented in the Oncomine database.
Data source and selection criteria (CBioPortal): The cBio Cancer Genomics Portal (http:/cbioportal.org), (open source cancer genomics data platform created by Memorial Sloan-Kettering Cancer Center (MSKCC)) was used to analyze the most common mutations of the selected genes in $\mathrm{PCa}$. The criteria used in order to include data sets in our analysis were the following: (1) type of cancer: $\mathrm{PCa}$; (2) the study must be published and (3) the study must consist of $>60$ samples.

Immunofluorescence experiments and quantitative microscopy. PC3 cells were fixed with $8 \%$ paraformaldehyde (PFA) ( $20 \mathrm{~min}$, room temperature) and stained with rhodamine-phalloidin ( $1 \mathrm{~h}$, room temperature). Confocal images were acquired by confocal microscopy (FV1000, Olympus, Tokyo, Japan) using an UPlanSApo 60x oil immersion objective (NA 1/41.35; Olympus), a diode laser of $543 \mathrm{~nm}$ as the excitation source and fluorescence was collected in the range of $555-655 \mathrm{~nm}$. We selected the regions from which filopodia from two neighboring cells were in contact, considered as 'contacts', and divided these regions into segments where the distance between the cells remained constant. An intensity profile for each of these areas was analyzed with a custom made Matlab algorithm to count contacts. A 'contact density' was defined for each region as the ratio between the number of contacts and the length of the profile.

To determine the distance to the first neighbor, cells were fixed with PFA8\% and stained with C-Laurdan. Wide field fluorescence images were acquired using an Olympus IX71 inverted epifluorescence microscope, with an UPlanSApo 10x objective (NA 0.30, Olympus). The images were obtained using a Qimaging EXI Aqua camera. The images were analyzed with an algorithm designed to binnarize the image and assign a label to each set of connected pixels. Every set of connected pixels represents a single cell in most cases and a cell cluster when the binnarization process could not distinguish among cells in contact or too close to each other. For every cell-cell cluster, the distance to all the other cell-cell clusters was calculated and to the first neighbor cell. Distances were normalized to the average cell radius.

Microtubule mechanics in PC3 cells was evaluated using confocal microscopy. Cells were treated with a primary antibody against tubulin and secondary antibody conjugated with Alexa-647. The effective Lp of microtubules in PC3 cells was determined using the methodology described by Gittes et al., ${ }^{37}$ for cells treated or not with hemin. Briefly, microtubule xy positions were recovered using a filament tracking routine, ${ }^{38}$ a Fourier analysis was performed on the shape of the filaments and mode amplitudes were recorded. Finally, $L p^{*}$ was obtained by fitting an inverse square law to the ensemble variance of these amplitudes as a function of the mode number.

Image processing for presentation: Confocal and wide field microscope images were processed for presentation using ImageJ software ( $\mathrm{NIH}$, Bethesda, MD, USA). Background of each channel was subtracted and in some cases a median filter (radius: 1 pixel) was applied only for presentation.

Immunoblotting: PC3 cells were lysed with Cellytic M Cell Lysis Reagent (Sigma-Aldrich, UK), incubated on ice for $20 \mathrm{~min}$, centrifuged at 12000 r.p.m. for $3 \mathrm{~min}$ and the supernatant was collected. Protein concentration was determined using the bicinchoninic acid (BCA) protein assay kit from (Sigma-Aldrich, UK). Samples were then resolved by SDS-PAGE, transferred to a nitrocellulose membrane (Invitrogen). Membranes were blocked for $1 \mathrm{~h}$ with $5 \%$ (w/v) non-fat milk

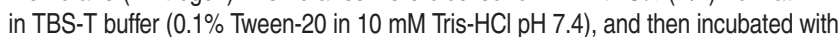

Figure 6 Analysis of RNAseq data of PCa cells overexpressing HO-1 pharmacologically or genetically. (a) RNAseq heat map of PC3 cells overexpressing HO-1 pharmacologically (Hemin) or genetically ( $\mathrm{PC} 3 \mathrm{HO}-1)$ and their respective controls. The transcripts were clustered in a conventional heat map analysis with red, blue and white plots representing transcripts with greater, intermediate and lower number of counts, respectively. (b) Schematic representation indicating the number and subset of upregulated $(\geqslant 2, \geqslant 3, \geqslant 5, \geqslant 8$ PC3HO-1-red circles; PC3 hemin-yellow circles) and downregulated genes ( $\leqslant-2, \leqslant-3, \leqslant-5, \leqslant-8, \mathrm{PC} 3 \mathrm{HO}-1$-green circles; $\mathrm{PC} 3$ hemin-blue circles). The circle intersections show the number of overlapping genes between both conditions. (c) Functional analyses of differentially expressed genes by DAVID (https://david.ncifcrf.gov/). GO analysis on twofold-regulated overlapping genes between both conditions. The resulting categories were further sub-clustered into two global categories named: 'cytoskeletal, cell adhesion and homeostasis categories' (yellow) and 'other function' (green). (d) Heat map for the twofold overlapping differentially expressed genes for cytoskeletal-associated GO categories and other pathways relevant to cell adhesion. Red, blue and white plots represent transcripts with greater, intermediate and lower number of counts, respectively. Genes associated specifically with cell adhesion and cell-cell communication are marked in blue and light blue respectively. (e) Gene interaction network between the twofold overlapping HO-1 upregulated (red) and downregulated (blue) GO categories. Interconnectivity is depicted either by genetic interactions (green lines), physical interactions (red lines) or common pathways (turquoise lines) 

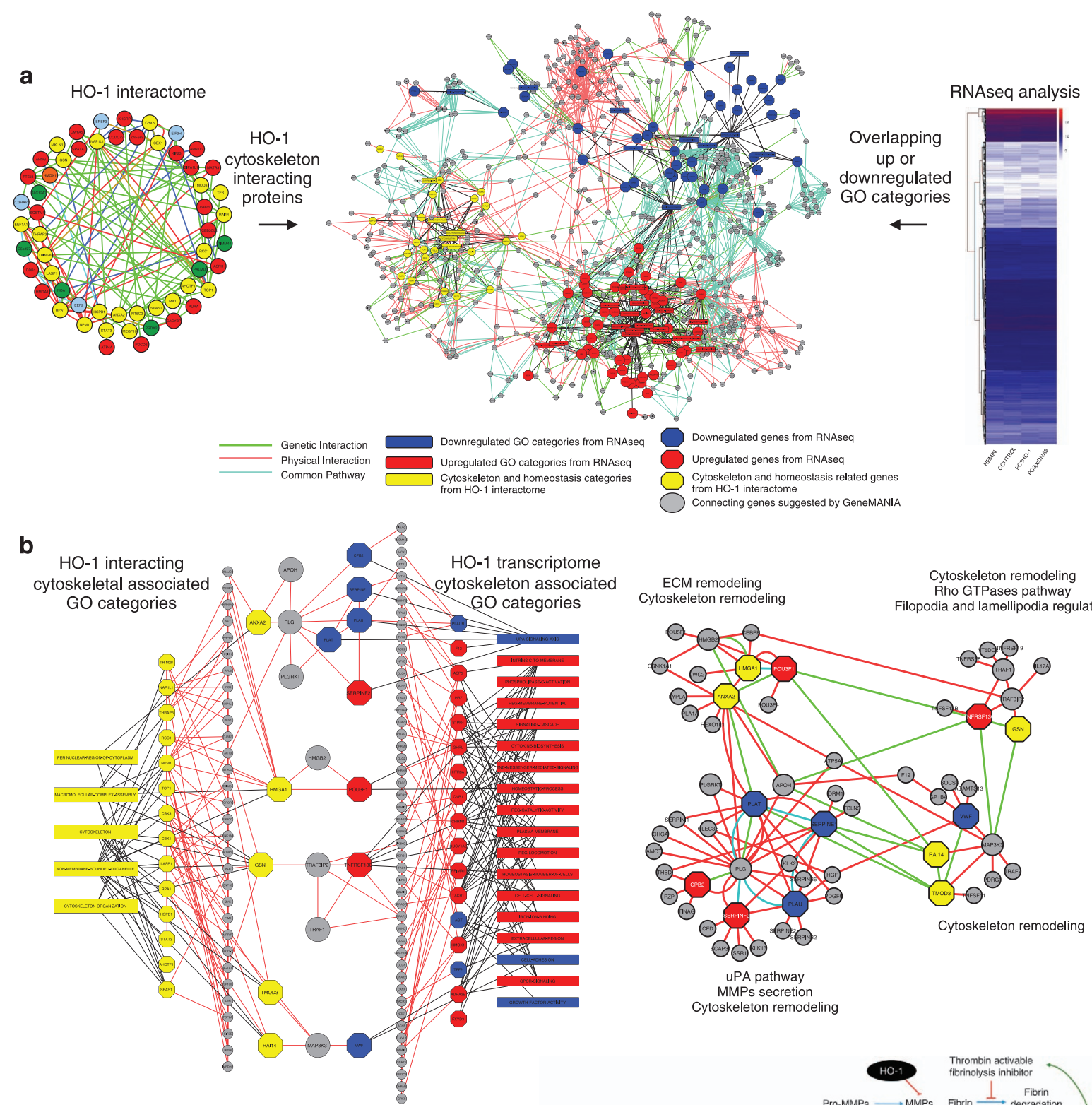

C

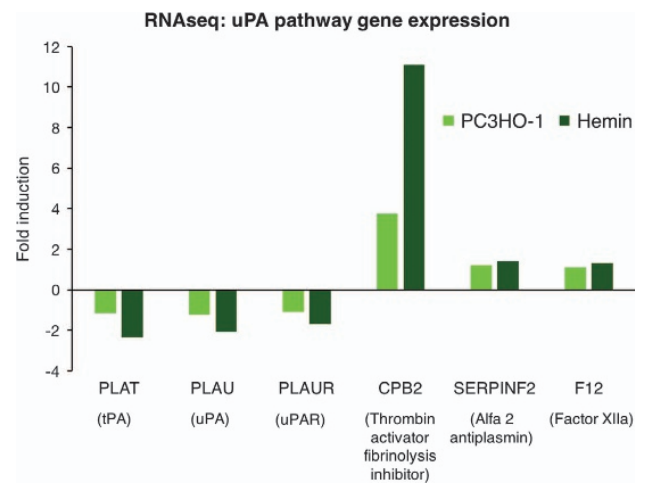

specific primary antibodies overnight at $4{ }^{\circ} \mathrm{C}$ : anti-HO-1 (1: 1000), anti- $\beta$-actin $(1: 4000)$. The next day, the membranes were incubated with secondary antibodies for $1 \mathrm{~h}$ at room temperature. Protein bands were detected using ECL reagents (Amersham Ltd).

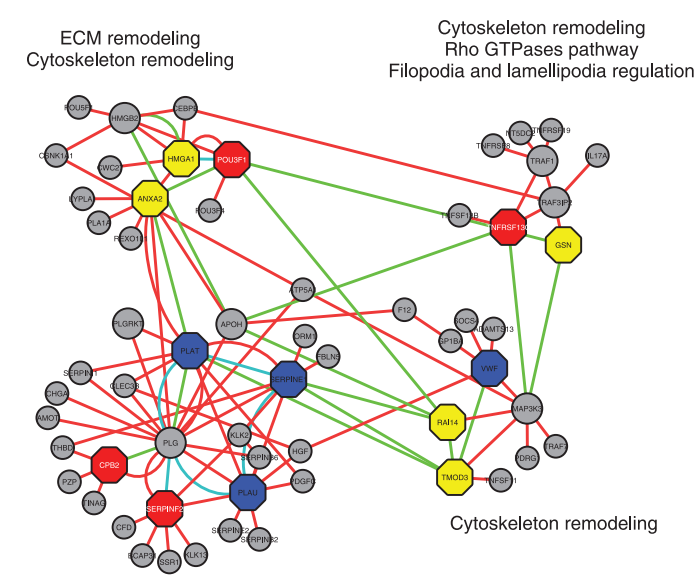

uPA pathway

Cytoskeleton remodeling

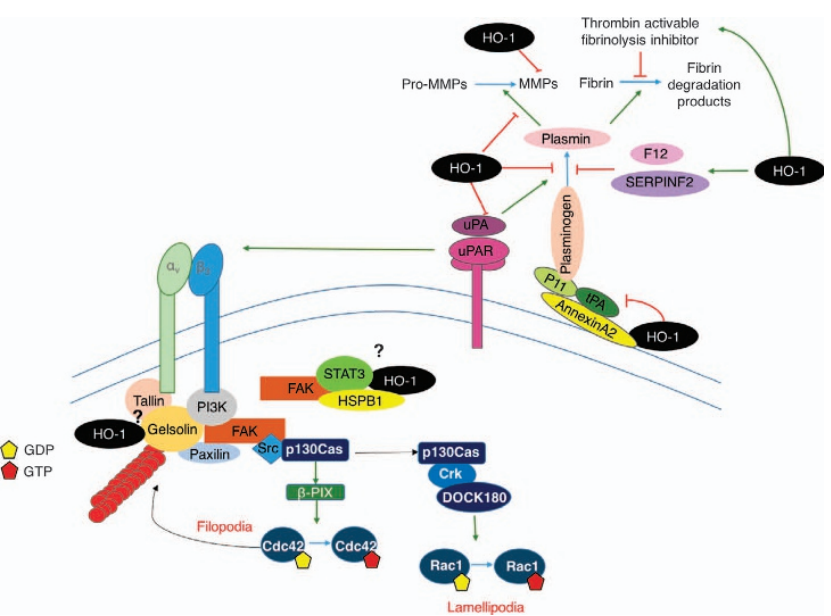

Cell tracking: Cells were seeded in $35 \mathrm{~mm}$ Petri dishes and cultured until confluence. The cells were then scraped with a $200 \mu$ l micropipette tip, denuding a strip of the monolayer and placed onto a Zeiss Axio Observer Microscope (Zeiss, Germany) equipped with an incubation chamber (ibidi Heating System, Ibidi, 
Figure 7 Integrated analysis of $\mathrm{HO}-1$ transcriptomic and proteomics data in $\mathrm{PCa}$. (a) Interaction network between proteins from cytoskeletal-related GO categories of the HO-1 interactome (yellow) and the twofold overlapping HO-1 upregulated (red) and downregulated (blue) GO categories from the RNAseq analysis. Interconnectivity is depicted either by genetic interactions (green lines), physical interactions (red lines) or common pathways (turquoise lines). (b) The above-mentioned interaction network was filtered by physical interconnectivity between proteins from cytoskeletal-related GO categories of the HO-1 interactome (yellow) and overlapping genes from the twofold upregulated (red) or downregulated (blue) cytoskeleton and homesotasis GO categories from the RNAseq analyses (left panel). Four nodes of connectivity between HO-1 interactome proteins and RNAseq data are displayed at the center of the figure (left panel) and expanded with there associated molecular and cellular pathways (right panel). (c) RNAseq expression profile for the urokinase-type plasminogen activator (UPA) pathway genes under HO-1 genetic (PC3HO-1- light green bars) or pharmacological (hemin - dark green bars) modulation (left panel). Schematic representation of the proposed model for the molecular pathways affected by HO-1 (right panel), resulting in impaired cell migration and invasion favoring a more adhesive and less aggressive phenotype in PCa. HO-1 overexpression impairs ECM degradation by downregulating MMPs, upregulating thrombin activable fibrinolysis inhibitor (TAFI), impairing fibrin degradation products. HO-1 also upregulates Alpha 2- anti-plasmin (SERPINF2), a serine protease inhibitor, responsible for inactivating plasmin, and downregulates UPA and urokinase receptor (UPAR). Downregulation of UPA/uPAR directly impacts on Rho GTPases pathways through the alpha V-beta 3 integrin receptor, which in turn affect lamellipodia and filopodia formation. HO-1 binds Gelsolin, STAT3 and HSPB1, potentially supporting its implication in lamellipodia and filopodia regulation

Germany) set at $37^{\circ} \mathrm{C}$. Phase contrast images were taken every 20 min during $48 \mathrm{~h}$ using a camera AxioCam HRm from Zeiss. The movies were processed using the cell tracking plug-in from Fiji (http://fiji.sc/Fiji) to extract cell trajectories and analyzed to obtain the MSD following equation 1 , where $x$ and $y$ are the coordinates of the particle, $t$ is time, and the brackets represent the trajectories ensamble average (see equation 1).

$M S D(t)=<(x(t)-x(0))^{\wedge} 2+(y(t)-y(0))^{\wedge} 2>$

Statistical analysis. Results are shown as mean \pm s.e.m of ' $n$ ' separate independent experiments unless otherwise is stated. In cases were binning was required, the size of the bins was determined by Freedman-Diaconi's rule. Boxplots show mean, median and whiskers represent the $5-95 \%$ window. For the distance analysis to the first neighboring cell, distributions were compared with a two-sample Kolmogorov-Smirnov test. A kernel smoothing density estimate was applied to the data in order to obtain probability density estimate curves.

RNA sequencing. The sequencing libraries for the RNA samples were prepared with the TruSeq Stranded Total RNA kit (Illumina Inc., San Diego, CA, USA), from $1 \mu \mathrm{g}$ total RNA. Following the manufacturer's instructions, the first step depleted rRNA from total RNA. After ribosomal depletion, the remaining RNA was purified, fragmented and primed for CDNA synthesis. Fragmented RNA was then reverse transcribed into first-strand CDNA using random primers. The next step removed the RNA template and synthesized a replacement strand, incorporating dUTP in place of dTTP to generate ds CDNA. AMPure XP beads were used to separate the ds CDNA from the second strand reaction mix resulting in blunt-ended cDNA. A single ' $A$ ' nucleotide was then added to the 3 ' ends of the blunt fragments. Multiple indexing adapters, containing a single ' $T$ ' nucleotide on the 3 ' end of the adapter, were ligated to the ends of the ds CDNA, preparing them for hybridization onto a flow cell. Libraries were purified and validated for appropriate size on a 2100 Bioanalyzer High Sensitivity DNA chip (Agilent Technologies, Inc., Santa Clara, CA, USA). The DNA library was quantitated using Kapa Biosystems qPCR kit (Wilmington, MA, USA), and then pooled together in an equimolar manner to a final concentration of $2 \mathrm{nM}$, following experimental design criteria. Each pool was denatured, diluted to $10 \mathrm{pM}$, and clustered to individual lanes of a HiSeq Flow Cell using an Illumina cBot and the corresponding paired-end TruSeq V3 cluster kit. Pooled, clustered samples were then run on a HiSeq2500 sequencer according to the manufacturer's recommended protocol (Illumina Inc.).

\section{Conflict of Interest}

The authors declare no conflict of interest.

Acknowledgements. We are grateful to the Prostate Cancer Foundation for the Young Investigator Award given to Geraldine Gueron. This work was supported by grants from the Prostate Cancer Foundation (Young Investigator award PCF), NIH-5P50-CA14038; Start-up grant from The University of North Carolina at Chapel Hill, AGENCIA-PICT 2013-0996 and 2015-1786 (Argentina), UBACyT 2014-2017 nº 497 (Argentina), and the National Cancer Institute (INC - Argentina).

\section{Author contributions}

Conception/design: EV and GG. Provision of reagents and facilities: PV, MS, LB, VL, $\mathrm{NN}, \mathrm{EV}$ and GG. Acquisition of data: AP, CP, FS, EO, NA and EL. Analysis/ interpretation of data: AP, CP, PV, JG, MM, JC, AW-R, LB, VL, NN, EV and GG. Writing of the manuscript: AP, EV and GG. Review of the manuscript: JG, AW-R, MM, JC, LB, VL and NN. Study supervision: EV and GG.

1. American Cancer Society. Cancer facts and figures. Cancer facts and figures, 2016. Available at http://www.cancer.org/research/cancerfactsstatisti.

2. Swami M. Proteomics: a discovery strategy for novel cancer biomarkers. Nat Rev Cancer 2010; 10: 597.

3. Clainche $\mathrm{C}$ Le, Carlier M-F. Regulation of actin assembly associated with protrusion and adhesion in cell migration. Physiol Rev 2008; 88: 489-513.

4. Okegawa T, Pong RC, Li Y, Hsieh JT. The role of cell adhesion molecule in cancer progression and its application in cancer therapy. Acta Biochim Polonica 2004; 51: 445-457.

5. Fan L, Wang H, Xia X, Rao Y, Ma X, Ma D et al. Loss of E-cadherin promotes prostate cancer metastasis via upregulation of metastasis-associated gene 1 expression. Oncol Lett 2012; 4: 1225-1233.

6. Parsons JT, Horwitz AR, Schwartz M A. Cell adhesion: integrating cytoskeletal dynamics and cellular tension. Nat Rev Mol Cell Biol 2010; 11: 633-643.

7. Bowser JL, Blackburn MR, Shipley GL, Molina JG, Dunner K Jr, Broaddus RR et al. Loss of CD73-mediated actin polymerization promotes endometrial tumor progression. J Clin Invest 2016; 126: 220-238.

8. Vasioukhin V, Bauer C, Yin M, Fuchs E. Directed actin polymerization is the driving force for epithelial cell-cell adhesion. Cell 2000; 100: 209-219.

9. Matsumoto $\mathrm{H}$, Ishikawa $\mathrm{K}$, Itabe $\mathrm{H}$, Maruyama $\mathrm{Y}$. Carbon monoxide and bilirubin from heme oxygenase-1 suppresses reactive oxygen species generation and plasminogen activator inhibitor-1 induction. Mol Cell Biochem 2006; 291: 21-28.

10. Otterbein LE, Soares MP, Yamashita K, Bach FH. Heme oxygenase-1: unleashing the protective properties of heme. Trends Immunol 2003; 24: 449-455.

11. Keyse SM, Tyrrell RM. Heme oxygenase is the major $32-\mathrm{kDa}$ stress protein induced in human skin fibroblasts by UVA radiation, hydrogen peroxide, and sodium arsenite. Proc Natl Acad Sci USA 1989; 86: 99-103.

12. Jozkowicz A, Was H, Dulak J. Heme oxygenase-1 in tumors: is it a false friend? Antioxidants Redox Signal 2007; 9: 2099-2117.

13. Gueron G, Giudice J, Valacco P, Paez A, Elguero B, Toscani M et al. Heme-oxygenase-1 implications in cell morphology and the adhesive behavior of prostate cancer cells. Oncotarget 2014; 5: 4087-4102.

14. Gueron G, De Siervi A, Ferrando M, Salierno M, De Luca P, Elguero B et al. Critical role of endogenous heme oxygenase 1 as a tuner of the invasive potential of prostate cancer cells. Mol Cancer Res 2009; 7: 1745-1755.

15. Ferrando M, Gueron G, Elguero B, Giudice J, Salles A, Leskow FC et al. Heme oxygenase 1 (HO-1) challenges the angiogenic switch in prostate cancer. Angiogenesis 2011; 14: 467-479.

16. Sudo $H$, Kodama $H A$, Amagai $Y$, Yamamoto $S$, Kasai $S$. In vitro differentiation and calcification in a new clonal osteogenic cell line derived from newborn mouse calvaria. J Cell Biol 1983; 96: 191-198.

17. Smith HW, Marra P, Marshall CJ. uPAR promotes formation of the p130Cas-Crk complex to activate Rac through DOCK180. J Cell Biol 2008; 182: 777-790.

18. An L, Liu CT, Qin XB, Liu QH, Liu Y, Yu SY. Protective effects of hemin in an experimental model of ventilator-induced lung injury. Eur J Pharmacol 2011; 661: 102-108.

19. Chen $C$, Zhang F, Zhang Z, Peng M, Wang $Y$, Chen $Y$ et al. TLR4 signaling-induced heme oxygenase upregulation in the acute lung injury: role in hemorrhagic shock and two-hit induced lung inflammation. Mol Biol Rep 2013; 40: 1167-1172.

20. Elguero B, Gueron G, Giudice J, Toscani MA, De Luca P, Zalazar F et al. Unveiling the association of STAT3 and HO-1 in prostate cancer: role beyond heme degradation. Neoplasia (New York, NY) 2012; 14: 1043-1056.

21. Lin C-W, Shen SC, Hou WC, Yang LY, Chen YC. Heme oxygenase-1 inhibits breast cancer invasion via suppressing the expression of matrix metalloproteinase-9. Mol Cancer Ther 2008; 7: 1195-1206.

22. Skrzypek K, Tertil M, Golda S, Ciesla M, Weglarczyk K, Collet G et al. Interplay between heme oxygenase-1 and miR-378 affects non-small cell lung carcinoma growth, vascularization, and metastasis. Antioxidants Redox Signal 2013; 19: 644-660. 
23. Tertil M, Golda S, Skrzypek K, Florczyk U, Weglarczyk K, Kotlinowski J et al. Nrf2-heme oxygenase-1 axis in mucoepidermoid carcinoma of the lung: Antitumoral effects associated with down-regulation of matrix metalloproteinases. Free Radical Biol Med 2015; 89 . 147-157.

24. Was H, Dulak J, Jozkowicz A. Heme oxygenase-1 in tumor biology and therapy. Curr Drug Targets 2010; 11: 1551-1570.

25. Wegiel B, Gallo D, Csizmadia E, Harris C, Belcher J, Vercellotti GM et al. Carbon monoxide expedites metabolic exhaustion to inhibit tumor growth. Cancer Res 2013; 73 7009-7021.

26. Stevenson RP, Veltman D, Machesky LM. Actin-bundling proteins in cancer progression at a glance. J Cell Sci 2012; 125: 1073-1079.

27. Narumiya S, Tanji M, Ishizaki T. Rho signaling, ROCK and mDia1, in transformation, metastasis and invasion. Cancer Metastasis Rev 2009; 28: 65-76.

28. Swaminathan V, Mythreye K, O'Brien ET, Berchuck A, Blobe GC, Superfine R. Mechanical Stiffness grades metastatic potential in patient tumor cells and in cancer cell lines. Cancer Res 2011; 71: 5075-5080.

29. Mohamedali KA, Li ZG, Starbuck MW, Wan X, Yang J, Kim S et al. Inhibition of prostate cancer osteoblastic progression with VEGF 121/rGEL, a single agent targeting osteoblasts, osteoclasts, and tumor neovasculature. Clin Cancer Res 2011; 17: 2328-2338.

30. Ferrando M, Wan X, Meiss R, Yang J, De Siervi A, Navone $\mathrm{N}$ et al. Heme oxygenase-1 $(\mathrm{HO}-1)$ expression in prostate cancer cells modulates the oxidative response in bone cells. PLoS One 2013; 8.

31. Daves MH, Hilsenbeck SG, Lau CC, Man TK. Meta-analysis of multiple microarray datasets reveals a common gene signature of metastasis in solid tumors. BMC Med Genomics 2011 4: 56.

32. Grasso CS, Wu YM, Robinson DR, Cao X, Dhanasekaran SM, Khan AP et al. The mutational landscape of lethal castration-resistant prostate cancer. Nature 2012; 487 239-243.

33. Griffith E, Coutts AS, Black DM. RNAi knockdown of the focal adhesion protein TES reveals its role in actin stress fibre organisation. Cell Motility Cytoskeleton 2005; 60 : 140-152.
34. Gunduz E, Gunduz M, Beder L, Nagatsuka H, Fukushima K, Sutcu R et al. Downregulation of TESTIN and its association with cancer history and a tendency toward poor survival in head and neck squamous cell carcinoma. Arch Otolaryngol Head Neck Surg 2009; 135: 254-260.

35. Valiyaveettil M, Bentley AA, Gursahaney P, Hussien R, Chakravarti R, Kureishy N et al. Novel role of the muskelin-RanBP9 complex as a nucleocytoplasmic mediator of cell morphology regulation. J Cell Biol 2008; 182: 727-739.

36. Keller A, Nesvizhskii Al, Kolker E, Aebersold R. Empirical statistical model to estimate the accuracy of peptide identifications made by MS/MS and database search. Analyt Chem 2002; 74: 5383-5392.

37. Gittes F, Mickey B, Nettleton J, Howard J. Flexural rigidity of microtubules and actin filaments measured from thermal fluctuations in shape. J Cell Biol 1993; 120: 923-934.

38. Pallavicini C, Levi V, Wetzler DE, Angiolini JF, Benseñor L, Despósito MA et al. Lateral motion and bending of microtubules studied with a new single-filament tracking routine in living cells. Biophys J 2014; 106: 2625-2635.

(c) Cell Death and Disease is an open-access journal published by Nature Publishing Group. This work is

licensed under a Creative Commons Attribution 4.0 International License. The images or other third party material in this article are included in the article's Creative Commons license, unless indicated otherwise in the credit line; if the material is not included under the Creative Commons license, users will need to obtain permission from the license holder to reproduce the material. To view a copy of this license, visit http://creativecommons.org/licenses/by/4.0/

(C) The Author(s) 2016

Supplementary Information accompanies this paper on Cell Death and Disease website (http://www.nature.com/cddis) 